\title{
Article \\ A Case Study for Decentralized Heat Storage Solutions in the Agroindustry Sector Using Phase Change Materials
}

\author{
Carlos Simão ${ }^{1,2}$, João Murta-Pina ${ }^{2,3, *}$, João Pedro Oliveira ${ }^{2,3}$, Luís Coelho ${ }^{4,5}{ }^{(}$, João Pássaro ${ }^{4,5}$, \\ Diogo Ferreira ${ }^{2}$, Fernando Reboredo ${ }^{2,6} \oplus^{\mathbb{D}}$, Tiago Jorge ${ }^{7}$ and Pedro Figueiredo ${ }^{8}$
}

1 CQS Unipessoal, Lda, Largo São Sebastião 44, 1050-205 Lisboa, Portugal; csimao@h1id.pt

2 NOVA School of Science and Technology (FCT NOVA), Campus da FCT NOVA, 2829-516 Caparica, Portugal; jpao@fct.unl.pt (J.P.O.); dadj.ferreira@campus.fct.unl.pt (D.F.); fhr@fct.unl.pt (F.R.)

3 Centre of Technology and Systems (CTS-UNINOVA), Campus da FCT NOVA, 2829-516 Caparica, Portugal

4 Polytechnic Institute of Setúbal, Campus do IPS, Estefanilha, 2910-761 Setúbal, Portugal;

luis.coelho@estsetubal.ips.pt (L.C.); joao.passaro@estsetubal.ips.pt (J.P.)

5 Centre for Energy and Environment Research (CINEA), Campus do IPS, Estefanilha, 2910-761 Setúbal, Portugal

6 Geobiosciences, Geotechnologies and Geoengineering (GeoBioTec), Campus da FCT NOVA, 2829-516 Caparica, Portugal

7 Metalogonde-Indústria Metalomecânica, Lda, Zona Industrial No. 2, 3720-069 Loureiro, Portugal; tiago.jorge@metalogonde.com

8 Transportes Figueiredo \& Figueiredo, Lda, Rua Pereiro 1150, 3700-743 Milheirós de Poiares, Portugal; pedro.figueiredo@tffigueiredo.pt

* Correspondence: jmmp@fct.unl.pt

check for

updates

Citation: Simão, C.; Murta-Pina, J.; Oliveira, J.P.; Coelho, L.; Pássaro, J.; Ferreira, D.; Reboredo, F.; Jorge, T.; Figueiredo, P. A Case Study for Decentralized Heat Storage Solutions in the Agroindustry Sector Using Phase Change Materials. AgriEngineering 2022, 4, 255-278. https://doi.org/10.3390/ agriengineering 4010018

Academic Editor: Piernicola Masella

Received: 23 January 2022

Accepted: 22 February 2022

Published: 28 February 2022

Publisher's Note: MDPI stays neutral with regard to jurisdictional claims in published maps and institutional affiliations.

Copyright: (C) 2022 by the authors. Licensee MDPI, Basel, Switzerland. This article is an open access article distributed under the terms and conditions of the Creative Commons Attribution (CC BY) license (https:// creativecommons.org/licenses/by/ $4.0 /)$.

\begin{abstract}
The development of thermal energy storage solutions (TES) in agroindustry allows reduction of production costs and improvement of operation sustainability. Such solutions require high storage capacity and the ability to adapt to existing equipment. The use of phase change materials (PCMs), which are able to store thermal energy as latent heat, creates new opportunities for heat storage solutions (LHS, latent heat storage) with higher energy density and improved performance when compared to sensible heat storage. New architectures are envisaged where heat storage is distributed throughout the production chain, creating prospects for the integration of renewable generation and recovery of industrial heat waste. This work aims to investigate the benefits of decentralized thermal storage architecture, directly incorporating PCM into the existing equipment of an agroindustry production line. To assess the feasibility and potential gain in the adoption of this TES/LHS distributed solution, a tempering and mixing equipment for food granules is selected as a case study, representing a larger cluster operating under the operation paradigm of water jacket heating. The behavior of the equipment, incorporating an inorganic PCM, is modeled and analyzed in the ANSYS Fluent software. Subsequently, a prototype is instrumented and used in laboratory tests, allowing for data collection and validation of the simulation model. This case study presents a demonstration of the increase in storage capacity and the extension of the discharge process when compared to a conventional solution that uses water for sensible heat storage.
\end{abstract}

Keywords: agroindustry; food processing; latent heat; phase change material (PCM); thermal energy storage

\section{Introduction}

Although there is no widely adopted definition for Agroindustry, The United Nations Industrial Development Organization provides one that suits several cases found in the literature, namely the "post-harvest activities involved in the transformation, preservation, and preparation of agricultural products for intermediate or final consumption" [1]. Agroindustry is related to commercializing and adding value to agricultural products, as well as 
to the links between enterprises and the supply chains for developing, transforming, and distributing those products [2]. Nevertheless, agroindustry is not limited to agriculture.

\subsection{Energy Consumption in Agroindustry}

Although agroindustrial plants are not seen as intensive energy consumers, the scale of agroindustry makes it responsible for a major fraction of worldwide energy consumption [3]. According to the Food and Agriculture Organization (FAO) from the United Nations, agriculture and agroindustry systems are characterized by a high dependence on energy, particularly fossil fuels, accounting for around $30 \%$ of worldwide energy consumption and $22 \%$ of greenhouse gas emissions (GHG) [4]. Specifically, for the food sector, FAO envisages a transition to energy-smart food systems as a solution to make them energy sustainable, with low GHG emissions, more robustness in the face of energy price variations, and able to contri e to food safety and sustainable development. This can be achieved by simultaneous action in distinct but strongly interlinked areas, namely energy efficiency, renewable energy integration, and circular economy.

Energy dependence in the agroindustry runs from the production of the raw material to transportation, processing, packaging, and storage of products, and extends to support activities, such as lighting or climatization [5]. In electrical or thermal energy form, the consumption pattern among companies in the agroindustrial sector varies significantly, depending on the products and performed operations, technological maturity, and access to combustible materials [5]. Although in some activities it is possible to observe the predominance of one or the other of the two sources, electrical energy has the advantage of being easily converted into thermal energy, reducing the dependence on fuels. In cases where combustion is used for the production of heat, its application is mainly aimed at the production of steam and hot water [6].

The per capita energy consumption and GHG emissions in the European Union in 2013 are shown in Figure 1, broken down into the food production stages. The largest consumer and polluter in the agroindustry value chain is agriculture, including forestry and fisheries, with around $33 \%$ of consumption and $67 \%$ of emissions. The environmental impact and the energy weighting in the cost of final products make energy efficiency a concern for the agroindustry operating models, which seek to enhance the integration of local renewable energy production, often in self-consumption schemes, in addition to the conventional energy sources. This however still presents various degrees of inefficiencies and challenges [7].

When integrated with renewable production solutions, the importance of storing energy surplus has promoted the study and development of multiple solutions, such as thermochemical storage, in which the interaction between different compounds is promoted, having the release of heat as a by-product [8]. Storage solutions show different levels of efficiency and performance, as well as the logistical requirements of space and volume. Often these solutions are suited to centralized storage schemes, requiring considerable amounts of exchanged energy to validate their economic viability.

\subsection{Thermal Energy Storage in Agroindustry}

Heat management is a key element in the treatment and transformation of food raw materials, thus essential to an industry that generally uses water as a medium for thermal energy storage (TES) in the form of sensible heat storage (SHS). When compared to other materials, water has the advantage of low cost and high availability. Water is also inert, and has therefore no risk of toxicity or corrosion [9]. The main drawbacks in SHS are the required temperature increase and volume of the storage medium, which can be relatively large, because of its thermal density, which can be relatively low.

An alternative to SHS solutions is latent heat storage (LHS), where energy is stored through the phase change of a medium at a constant temperature. The materials used in these solutions are called Phase Change Materials (PCMs). In the agroindustry sector, typically organic and inorganic PCMs [10] are used (although there are also materials of the 
eutectic type [11], they are seldom used due to their high cost and low latent and specific heat, among other factors [12]). They are characterized by having high enthalpy, which allows them to store high amounts of thermal energy in small volumes when compared to SHS. Through the adequation of their phase change temperatures, these materials can be successfully integrated into the operations of the agroindustry sector, increasing the installed thermal energy storage capacity and promoting the stability of the operating temperatures. Simultaneously, since agroindustry is a major user of low-temperature heat, i.e., below $120^{\circ} \mathrm{C}$, decentralized TES/LHS solutions represent an opportunity for new approaches to energy efficiency, namely through integrating renewable energy production, as well as recovering and valuing industrial waste heat (IWH) beyond that already commonly used [6].

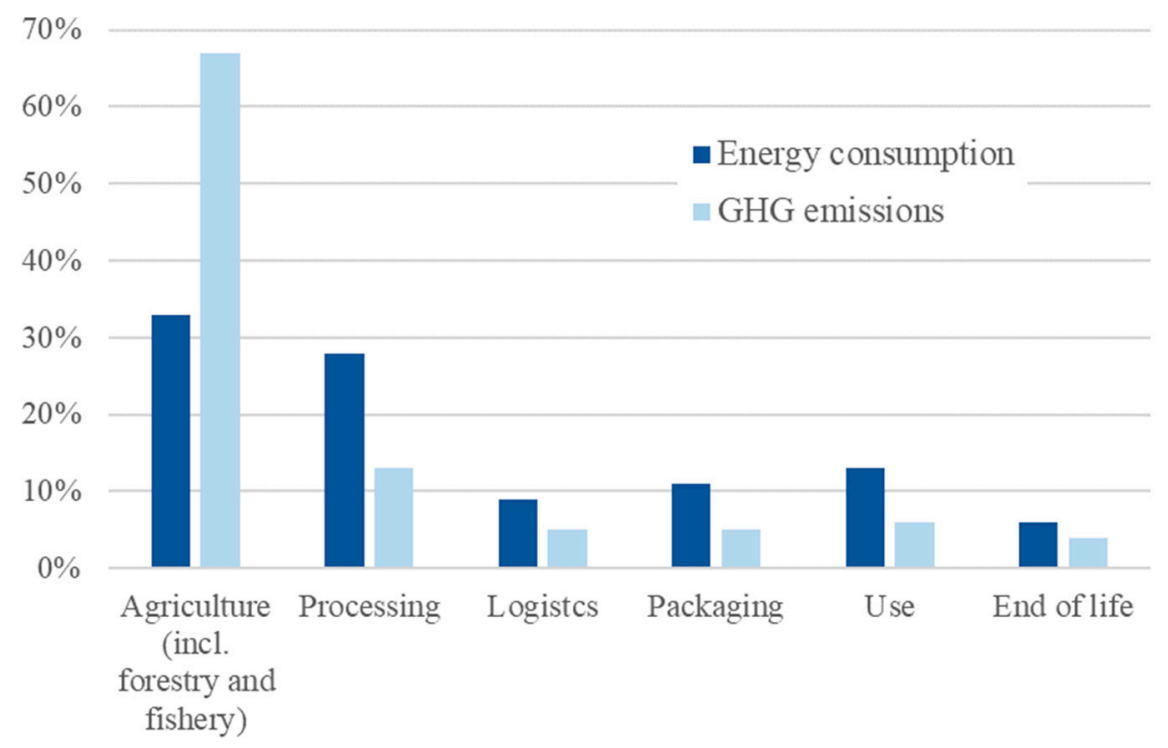

Figure 1. Per capita energy consumption (average 23.6 GJ) and GHG emissions (2965 kg of $\left.\mathrm{CO}_{2}\right)$ in the food system in the EU in 2013, broken down into food production stages. Adapted from [13].

\subsection{TES: Centralized SHS vs. Decentralized LHS}

In agroindustry, SHS using water tanks heated with renewable energy surplus represents the state of the art and technological maturity of TES solutions, and suffers from all the inefficiencies previously mentioned, namely, requiring relatively high storage volumes in centralized schemes that do not allow the addressing of temperature stabilization in the storage medium, often with relevant losses due to heat radiation. In addition to this, there is also the stiffness of the solution, the inability to address the dynamics and flexibility of the production lines, and the inability to recover smaller heat losses, which are wasted along the production chain. A scheme based on centralized SHS integrating renewables (solar thermal) is represented in Figure 2.

If it is considered that most processing operations in the agroindustry sector require some form of heat for the transformation and preservation of food, the possibility of storing thermal energy in the form of latent heat in a decentralized model integrated along the production lines, presents several potential operational advantages:

- $\quad$ By storing energy in the form of latent heat, the passive stabilization of the operating temperature is enhanced, minimizing the risks of food degradation and consequently improving the quality of the final products;

- The possibility of distributed storage incorporated into the production equipment provides energy flexibility for the electrical grid and reduces the logistics, space, and infrastructure requirements inherent to centralized storage in the form of sensible heat;

- It directly addresses the problem of reducing or recovering energy losses associated with IWH along the production lines. 


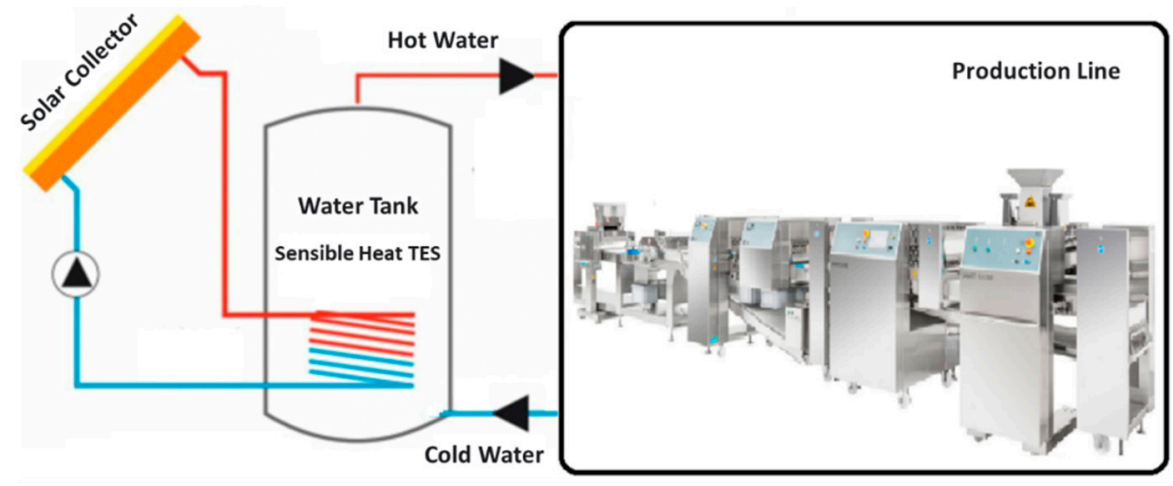

Figure 2. Diagram of a sensible-heat centralized TES solution, with solar energy integration.

Such a decentralized scheme is represented in Figure 3, where energy storage is envisaged by PCMs.

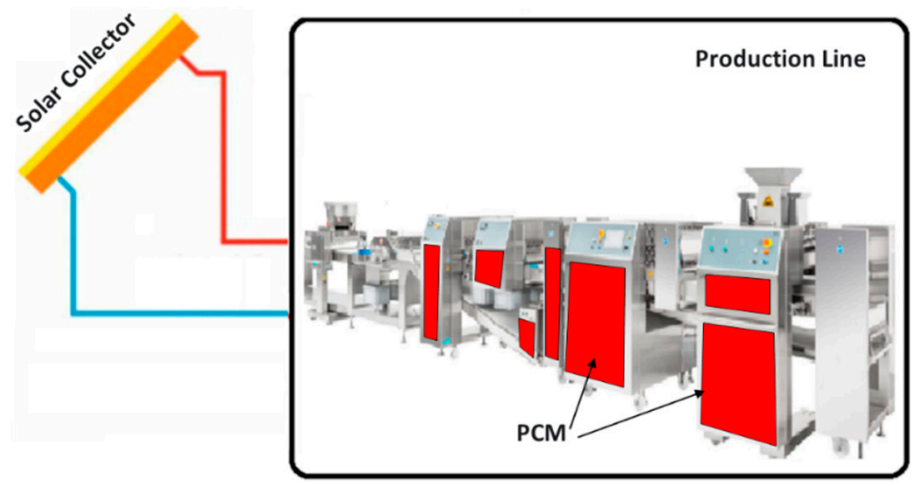

Figure 3. Diagram of a latent-heat decentralized TES solution, with solar energy integration. PCMs provide the distributed storage medium.

The main obstacles to a solution of this nature are the following:

- The high investment required in the existing technology park, whose exploitation longevity extends for several years, preventing simple replacement by a new technological generation;

- The risk of introducing materials that may be toxic, corrosive, and/or flammable, in an industry that demands the highest safety criteria. For this to be feasible, encapsulation solutions are needed to ensure operation safety, also without compromising the heat exchange capacity necessary for the energy viability of the solution, at an affordable cost.

Therefore, it is important to demonstrate the possibility of introducing PCM materials in agroindustry production chains as an efficient thermal energy storage solution, without requiring the replacement of the existing technological park or a significant change in operational and maintenance procedures, and also assuring safety and quality of products.

\subsection{Research Goals}

This work aims to explore and evaluate the possibility of improving the energy efficiency in agroindustry operations while providing grid flexibility, using PCMs in a distributed TES architecture, by directly integrating them into the production equipment with minimal operational impact.

To validate and demonstrate this concept, a class of equipment that operates on a water jacket basis, using the water bath technique, was selected. Among them, melting and mixing equipment for granulated materials (milk, chocolate, butter) was used as a case study for the distributed TES paradigm. 
To develop the methodologies and tools that allow scaling up of the use of PCMs as part of TES solutions for agroindustry, and applying them in the modeling of real case scenarios, ANSYS Fluent software was first used for computational fluid dynamics simulations of the performance of an inorganic PCM incorporated into the selected equipment. Subsequent experiments allowed comparison of the main aspects of the storage capacity and charge/discharge processes of the proposed system with a conventional SHS solution using water.

\section{Materials and Methods}

\subsection{Test Equipment and Case Study}

The equipment used in this work was a tempering and mixing machine to melt granules of chocolate mixed with milk. Figure 4 shows the machine, which can be segmented into two functional components:

- A 23 L inner cylindrical tank, where food granules are deposited and heated, and melting and mixing operations are carried out using a 7-rpm agitator arm;

- A 38 L outer cylindrical tank, heated at the base by a $3000 \mathrm{~W}$ heat source (electrical resistor).
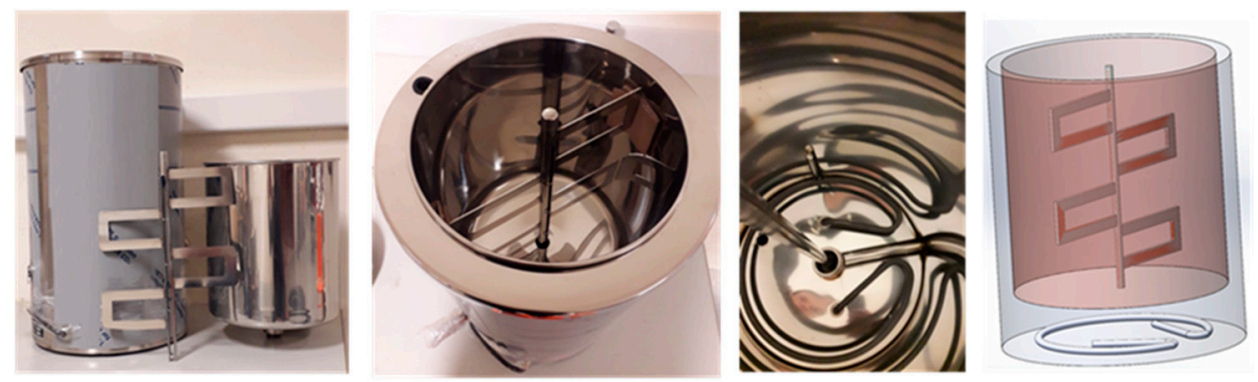

Figure 4. Tempering and mixing equipment for granulated materials (e.g., milk, chocolate, or butter). From left to right: outer tank, rotating arm, and inner tanks; top view of the equipment; heating resistor; computer view of the equipment.

Both tanks are made of A316 steel. The volume between the outer and inner tanks is filled with water, which works as a thermal storage medium for transmission and homogenization of the heat produced in the electrical resistor at the base. This volume of water $(11.5 \mathrm{~L})$ will be partially replaced by a PCM to increase the energy storage capacity of the equipment, maintaining the temperature of the food product over the processing cycle ( 8 to $12 \mathrm{~h}$ ). During this period, the chocolate must remain liquefied at a stable temperature to maintain its organoleptic properties. Assuring this in the conventional operating model requires repeatedly turning the resistor on and off, with intermittent power consumption.

Several agroindustry processes operate at low temperatures (below $120^{\circ} \mathrm{C}$ ) [14], and their residual heat can be recovered as a source for this equipment. Nevertheless, electrical energy from the grid or renewable generation (as solar thermal or photovoltaics) can also be used to provide heat.

\subsection{Phase Change Materials (PCMs)}

The storage of thermal energy in the form of latent heat is provided by materials that change their thermodynamic state, usually between the solid and the liquid phases. During the phase change, the temperature remains constant, while the amount of energy stored at the molecular level is substantially increased, promoting that change. In the development of these materials, the challenge is to obtain substances able to store more energy in less mass at different and adjustable temperature levels, allowing their exploration in a wide range of applications [15] including construction, the automobile sectors, telecommunications, and medicine, among others [11,16]. 


\subsubsection{Main Properties of PCMs}

With a large spectrum of applications and no regulatory barriers of relevance, the exploitation of PCMs is conditioned by their availability and cost, and by a set of technical characteristics that dictate their use. Among the main properties, the following are highlighted [17]:

- $\quad$ Phase change temperature, $T_{p c}$;

- $\quad$ Latent heat per unit of volume or mass, $L$;

- Thermal conductivity, $k$;

- Chemical stability, to allow a high number of melting/solidification cycles [12] (longevity);

- Degree of corrosion, toxicity, and flammability, so that in the event of leakage, no toxic or dangerous substances are released to the environment, which in any way may compromise the operation and safety.

PCMs are classified into three major groups, namely organic, inorganic, and eutectic; see [10] for a detailed description. Encapsulation is often used in applications, since its use involves a liquid phase or contact with other elements [12]. PCMs are often available as powder, with dimensions ranging from microns to a few millimeters (micro-encapsulation), or in enclosures with dimensions in the order of centimeters (macro-encapsulation). When choosing encapsulation materials, it must be assured that they do not react with the PCM in case of deterioration over time, and that they do not affect the heat transfer capacity. The latter is also influenced by the encapsulation geometry, which is closely linked to the way that PCMs are integrated into the target system.

\subsubsection{Main Properties and Selection of the PCMs}

Organic PCMs of the paraffin type and in the form of inorganic hydrated salts are of special interest for most of the specificities and operating temperatures of agroindustrial processes. Their phase change temperatures usually lie in the food processing operation range. The former usually have a lower latent heat capacity and thermal conductivity, but are more stable than the latter, and although flammable, show a lower risk of corrosion and toxicity. These were determinant characteristics for the selection of the PCM to be used in the case study, but it was also important to consider the physical characteristics and geometry of the equipment selected for experimental testing and its influence on the encapsulation strategy. The possibility of a good encapsulation solution may justify the option of a hydrated salt, which would allow the greater energy storage capacity relevant in low volume scenarios. On the other hand, the possible risk of segregation, common in the encapsulation of salts [18], could justify the option of paraffin.

The PCM phase change temperature is one main criterion for selection, and this should be equal to the operating temperature of the equipment. In this case, paraffins and hydrated salts with $T_{p c}=34{ }^{\circ} \mathrm{C}$ (the same working temperature as for the tempering and mixture of chocolate granules) were first selected. In addition to the adequacy of the phase change temperature, the chosen PCM must have relatively high values of latent heat of fusion and density to provide a high thermal storage capacity in the available volume $[19,20]$. The thermal conductivity is also equally relevant in order to guarantee a good discharge capacity. Low risks of toxicity and corrosion are also mandatory parameters, particularly when dealing with food products.

In this context, it is fundamental to establish a decision criterion to guide the choice of the PCM, considering the multiplicity of parameters and possible risks. With this objective, a decision model was established integrating the parameters that were considered most relevant and which were clustered according to their thermal, mechanical, and chemical nature, allowing the proposal of criteria of adequacy and risk according to the nature of the targeted operation.

Over this clustering of parameters and risks, a valuation criteria matrix was developed, structured on three importance levels. The highest importance, 3, was attributed to exclusion criteria (melting temperature and cost), or those that had a direct impact on the performance (fusion enthalpy and segregation). The lowest importance, 1, was attributed 
to the risk criteria, which was justified by the possibility that these could be improved through an appropriate encapsulation solution. Intermediate criteria had importance 2. In total, each PCM could score up to 100 points.

Although there is still no information or a holistic model that considers the impact of the life cycle of the PCM, both in terms of materials and embodied energy, from production to end-of-life recycling and considering the encapsulation solution and the process of exploration [21], it was considered important to include a qualitative criterion associated with the known impacts of the recycling of different types of end-of-life PCMs.

The previous methodology is exemplified for two of the selected PCMs, a hydrated salt and a paraffin, in Figure 5. The hydrated salt was the reference S34 from PCM Products Company, whose thermophysical parameters are described in Table 1. This PCM had a thermal storage capacity superior to the paraffin, but its performance was worse in terms of segregation, corrosion, and toxicity. Nevertheless, the encapsulation of the material mitigate these impacts. Its lower material cost will be relevant in the eventual dissemination and massification of the results.

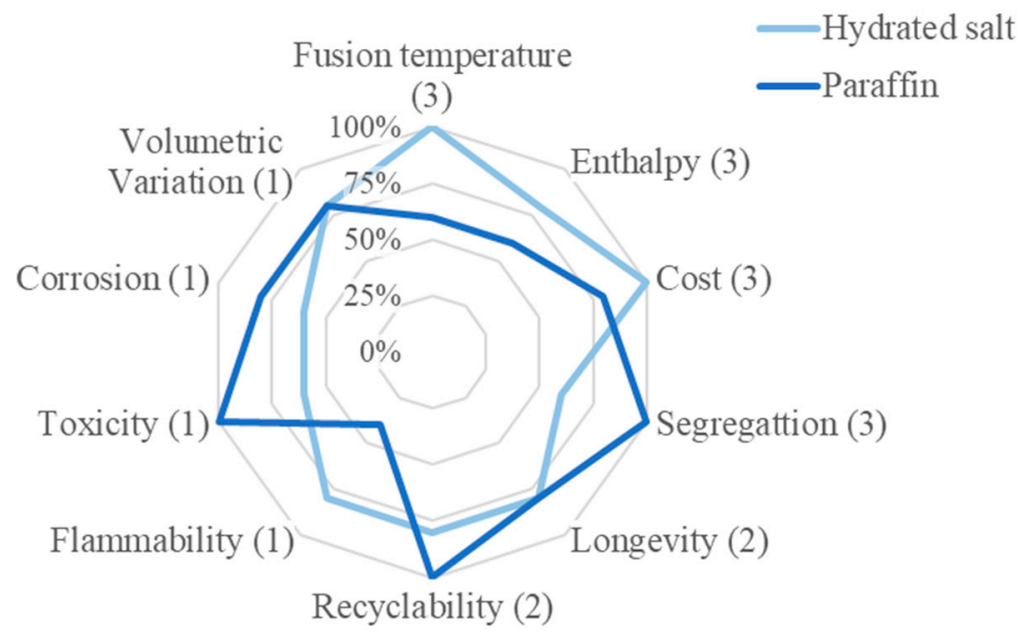

Figure 5. Application of the PCM selection methodology to two materials, a hydrated salt and a paraffin. The importance criteria are shown in brackets, for each of the selection parameters. The hydrated salt is better in three of these when compared to the paraffin. Scores are shown in percentage, given by the importance times adequacy. Thus, $100 \%$ will correspond to 15,10 , and 5 points, for the importance of 3, 2, and 1, respectively. The hydrated salt scored 81 and the paraffin 78.

Table 1. Thermophysical properties of the selected PCM, reference S34 from the PCM Products Company.

\begin{tabular}{cc}
\hline PCM Property & Value \\
\hline Phase change temperature, $T_{p c}$ & $34{ }^{\circ} \mathrm{C}$ \\
Density, $\rho$ & $2100 \mathrm{~kg} \mathrm{~m}^{3}$ \\
Specific heat, $C_{P}$ & $2.1 \mathrm{~kJ} \mathrm{~kg}^{-1} \mathrm{~K}^{-1}$ \\
Thermal conductivity, $k$ & $0.52 \mathrm{~W} \mathrm{~m}^{-1} \mathrm{~K}^{-1}$ \\
Dynamic viscosity, $\mu$ & $5.9 \mathrm{~kg} \mathrm{~m}^{-1} \mathrm{~s}^{-1}$ \\
Phase change enthalpy, $\Delta H_{m}$ & $140 \mathrm{~kJ} \mathrm{~kg}^{-1}$ \\
\hline
\end{tabular}

\subsubsection{PCM Encapsulation}

Three encapsulation options were initially considered for the selected S34 PCM, namely, spheres, rods, and tablets; see Figure 6. Each one was influenced by different variables of thermophysical and operational nature, and the encapsulation selection process aimed to establish decision criteria that would be replicable in other agroindustrial operations and equipment scenarios. In all solutions, to minimize changes in equipment and operational and maintenance processes, it was decided to maintain some of the water in the jacket, to act as an accelerator and homogenizer in the heat transfer process between the heat source, the PCM, and the inner tank. 

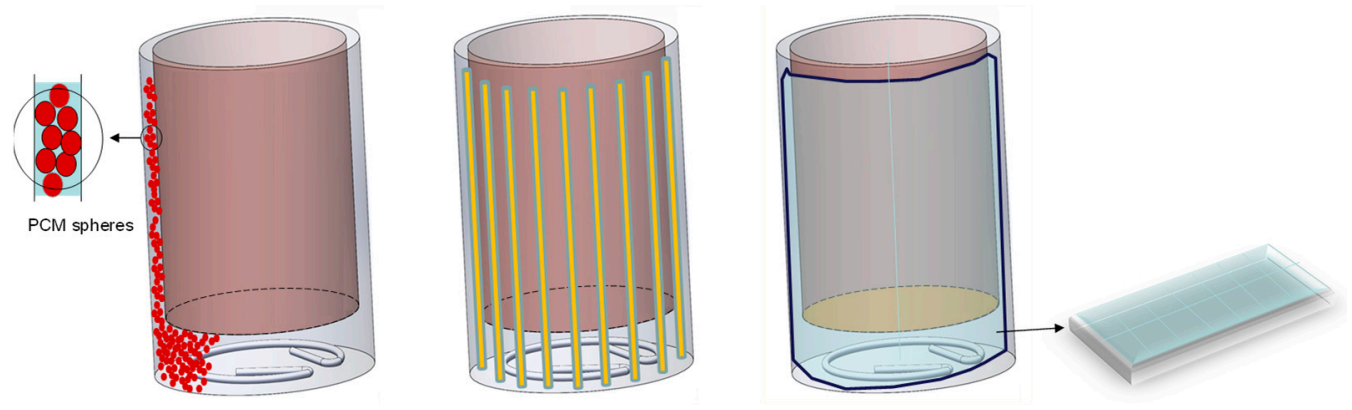

Figure 6. The PCM encapsulation options that were initially considered, namely, from left to right, spheres, rods, and tablets.

The spheres solution (around $1 \mathrm{~cm}$ diameter) offered the largest heat exchange surface [22], but there were operational and food security concerns, as the direct contact of the spheres with the heat source could compromise the involucre of the PCM and release toxic compounds. It would have to be ensured that the spheres would not physically contact the electric resistance at the base of the external tank, guaranteeing at the same time a straightforward collecting process during the cleaning procedures of the tanks.

The rods would be the easiest way to introduce a PCM in the water jacket, with minimal changes in maintenance procedures. However, vertical rods (with lengths over $5 \mathrm{~cm}$ ) raise segregation concerns, particularly when working with hydrated salt PCMs (these would be neglectable for the paraffin, see Figure 5).

The tablet solution showed the greatest capacity for including the required PCM volume (3.5 L) and consequently, the highest prospective stored energy without operation concerns, thus allowing for easier dissemination of outcomes, and was therefore selected. Nevertheless, it required a flexible encapsulation material, able to adjust to the tank shape during the phase changes (solid-liquid-solid), without damage risk.

The encapsulation material must also be carefully selected. Aluminum foil for tablets, copper for rods, and different plastic polymers for all the options were considered. Each of these had advantages and disadvantages. Plastic polymers allow for lower encapsulation costs, but they are prone to corrosion and mechanical wear. They also have a relatively low heat conductivity, which can compromise the charge/discharge efficiency of the PCM. The metallic encapsulation is more expensive, but offers higher conductivity and robustness than polymers. For the sake of simplicity, a plastic polymer material was selected for the encapsulation of the PCM tablets.

The selected solution was necessarily a trade-off that allowed for the carrying out of the experimental tests. Without prejudice to this choice or the experimental test results, it was later found (see Section 3.2.2) that the sealing of the packaging was compromised in some of the tablets (see Figure 7). This occurrence limits the applicability of this encapsulation material in real-world solutions, which will have a more intense and prolonged exploration and deserves further investigation. 

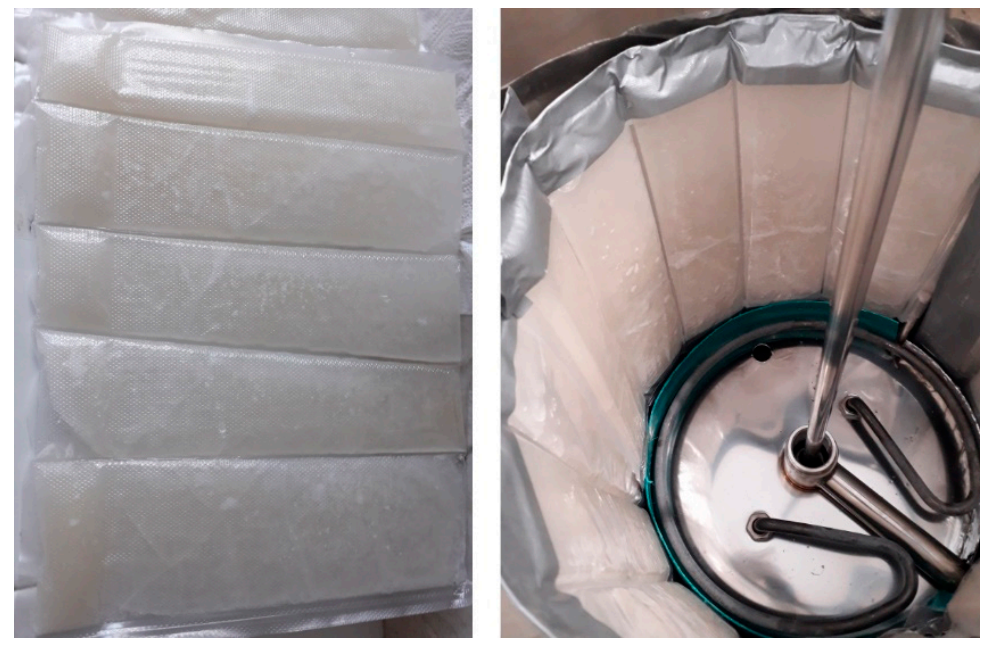

Figure 7. S34 PCM encapsulation, placed between the inner and outer tanks.

\section{Results}

\subsection{Simulation Results}

The ANSYS Fluent computational fluid dynamics (CFD) package was used for simulations that evaluate the thermal energy storage performance of the equipment operating either with water (SHS) or with PCMs (LHS), as well as the evolution of the main variables influencing the charge and the discharge processes. For this purpose, a model of the geometry of the equipment was developed.

In the first simulations, a 3D model with a mesh of more than three million elements was developed; see Figure 8. Simulations converged slowly, with more than three days of processing for each step of five seconds with an Intel Xeon W-2155 Processor $(3.30 \mathrm{GHz})$. To overcome this restriction, considering the symmetry of the equipment, a $2 \mathrm{D}$ cross-section model was developed; see Figure 9. With this solution, it was possible to improve the simulation speeds to approximately five minutes for steps of five seconds.

The grid and time-step independence study are reflected in Figure 10. Three grids were tested with $35 \times 10^{3}, 28 \times 10^{3}$, and $19 \times 10^{3}$ elements, using the PCM average mass temperature as a control variable. With a slight difference that excluded the $19 \times 10^{3}$ elements grid, the two others almost overlapped (over $98 \%$ convergence). The $28 \times 10^{3}$ elements were selected for all the numeric work, as the other would only increase the processing time with negligible improvement on the results.
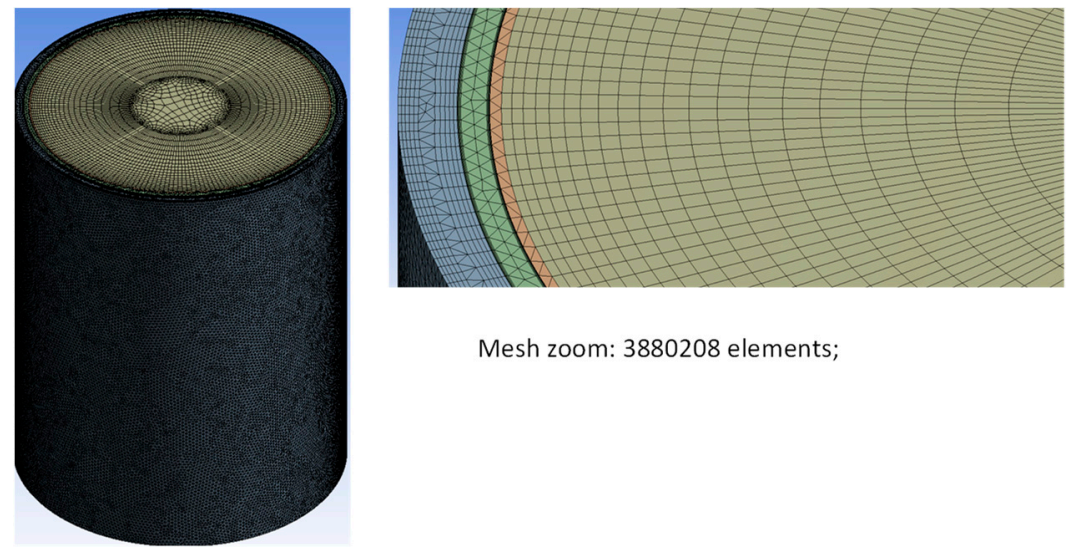

Mesh zoom: 3880208 elements;

Figure 8. 3D model and respective mesh in ANSYS Fluent. 

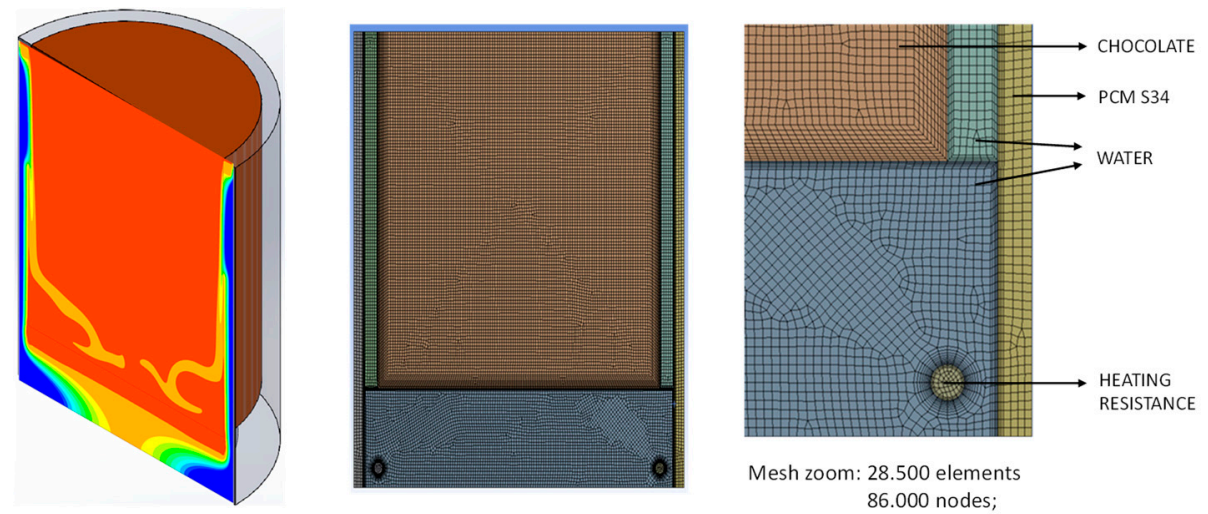

Figure 9. ANSYS Fluent 2D cross-section model and respective mesh.
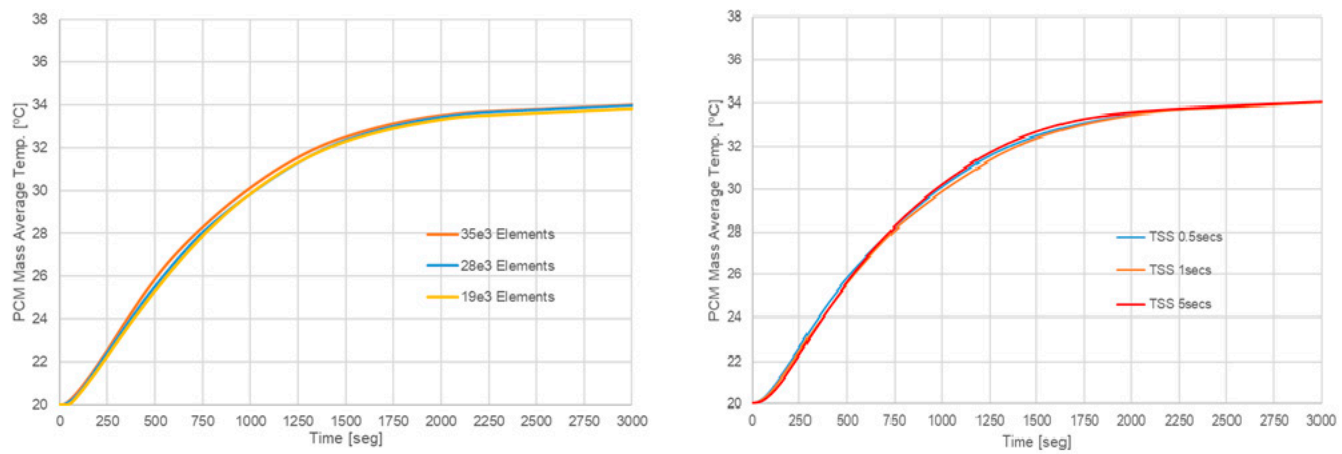

Figure 10. Grid independence study (right) and time step independence study (left).

In the time-step independence study, three step sizes were tested, namely, $0.5,1$, and $5 \mathrm{~s}$, with similar results. As such, the first $2000 \mathrm{~s}$ of the simulation were run with a $1 \mathrm{~s}$ time step, and the remaining duration with a time step of $5 \mathrm{~s}$. This ensured that the time frame where the temperatures had a greater gradient had a more refined time step, to ensure minimal solution inaccuracy. Later, the time step increased to speed up the simulation where there were smaller gradients.

The PCM phase change phenomenon was simulated by the enthalpy-porosity formulation, see below. The ability of this approach to model the process has been demonstrated in the past [23-25]. The complete numerical model solves the mass, momentum, and energy transport equations for constant density. The viscous dissipation term is considered negligible, while the energy equation is solved in the form of total enthalpy.

$$
\begin{gathered}
\frac{\partial \rho}{\partial t}+\nabla \cdot(\rho \vec{V})=0 \\
\frac{\partial \rho \vec{V}}{\partial t}+\nabla \cdot(\rho \vec{V} \vec{V})=-\nabla p+\mu \nabla^{2} \vec{V}+\rho_{o} \beta\left(T-T_{o}\right) \vec{g}+\vec{S} \\
\frac{\partial \rho H}{\partial t}+\nabla \cdot(\rho \vec{V} H)=\nabla \cdot(k \nabla T)
\end{gathered}
$$

In the previous equations, $V$ represents the velocity, $\rho$ the density, $\mu$ the dynamic viscosity, $k$ the thermal conductivity, $T$ the temperature, and $H$ the total enthalpy, defined as:

$$
H=h+\Delta H
$$

where

$$
h=h_{\text {ref }}+\int_{\text {Tref }}^{T} c_{p} d T,
$$


and $h$ represents the sensible enthalpy, $c_{p}$ is the specific heat, and $\Delta H$ is the phase change enthalpy. The temperature is calculated through the total enthalpy and the liquid mass fraction, $\gamma$, defined as:

$$
\begin{cases}\gamma=0 & \text { if } T<T_{\text {solid }} \\ \gamma=\frac{T-T_{\text {solid }}}{T_{\text {liquid }}-T_{\text {solid }}} & \text { if } T_{\text {solid }}<T<T_{\text {liquid }}, \\ \gamma=1 & \text { if } T>T_{\text {liquid }}\end{cases}
$$

where $T_{\text {solid }}$ is the solidification temperature and $T_{\text {liquid }}$ is the fusion temperature. Locally, the phase change enthalpy can be written in terms of the liquid mass fraction and the PCM latent heat, $L$, as, $\Delta H=\gamma L$. The enthalpy-porosity formulation treats different phases as porous media through the following source term, $\vec{S}$ :

$$
\vec{S}=\frac{(1-\gamma)^{2}}{\left(\gamma^{3}+\xi\right)} A_{m u s h y} \vec{V}
$$

In the previous equation, $A_{\text {mushy }}$ is the mushy zone constant, which describes how steeply the velocity is reduced to zero when the material solidifies. In the present study, a standard value of $A_{\text {mushy }}=10^{5}$ is employed. The constant $\xi$ is a small value, typically $10^{-3}$, introduced to prevent division by zero.

The effect of natural convection in the PCM was accounted for by the Boussinesq approximation [25].

Using the 2D CFD model, four transient thermal analysis cases were developed, considering the charge and discharge of SHS and/or LHS, thus using water or water + PCM, as TES mediums:

- Case 1-Charge: water jacket filled with water (11.5 L), no PCM, constant heating source $\left(38^{\circ} \mathrm{C}\right)$, inner tank partially filled $(60 \%)$ with chocolate, with the chocolate mix starting at ambient temperature $\left(20^{\circ} \mathrm{C}\right)$.

- $\quad$ Case 2-Discharge: starting at Case 1 fully charged and the chocolate mix fully melted at $34{ }^{\circ} \mathrm{C}$.

- $\quad$ Case 3-Charge: water jacket filled with 3.5 L of S34 and 8 L of water, constant heating source $\left(38{ }^{\circ} \mathrm{C}\right)$, inner tank partially filled $(60 \%)$ with the chocolate mix starting at ambient temperature $\left(20^{\circ} \mathrm{C}\right)$.

- $\quad$ Case 4-Discharge: starting at Case 3 fully charged and the chocolate mix fully melted at $34{ }^{\circ} \mathrm{C}$.

The characteristics of commercial chocolate with 55\% cocoa were used in the simulations. Its most relevant properties are described in Table 2.

Table 2. Chocolate properties used in simulations.

\begin{tabular}{cc}
\hline Chocolate Property & Value \\
\hline Density, $\rho$ & $1260 \mathrm{~kg} \mathrm{~m}^{3}$ \\
Specific heat, $C_{P}$ & $1.2 \mathrm{~kJ} \mathrm{~kg}^{-1} \mathrm{~K}^{-1}$ \\
Thermal conductivity, $k$ & $0.09 \mathrm{~W} \mathrm{~m}^{-1} \mathrm{~K}^{-1}$ \\
\hline
\end{tabular}

In each scenario, the water jacket charge and discharge and the chocolate temperature evolution were analyzed at two intervals, namely from 30 to $34{ }^{\circ} \mathrm{C}$ and from 34 to $38{ }^{\circ} \mathrm{C}$, seeking to assess:

1. The formation of horizontal and vertical temperature gradients.

2. The evolution of the liquid fraction of the chocolate mass.

3. The increase in the energy stored in the water jacket.

4. The increase in the operation period, during the PCM discharge. 
An ambient temperature of $20^{\circ} \mathrm{C}$ and an external convective heat transfer coefficient of $4.12 \mathrm{~W} \mathrm{~m}^{-2} \mathrm{~K}^{-1}$ were defined as boundary conditions.

Case 3 (charge of water + PCM) results are presented in the following, and these are compared with Case 1 (charge of water). The results for Case 3 are first shown in Figures 11 and 12.

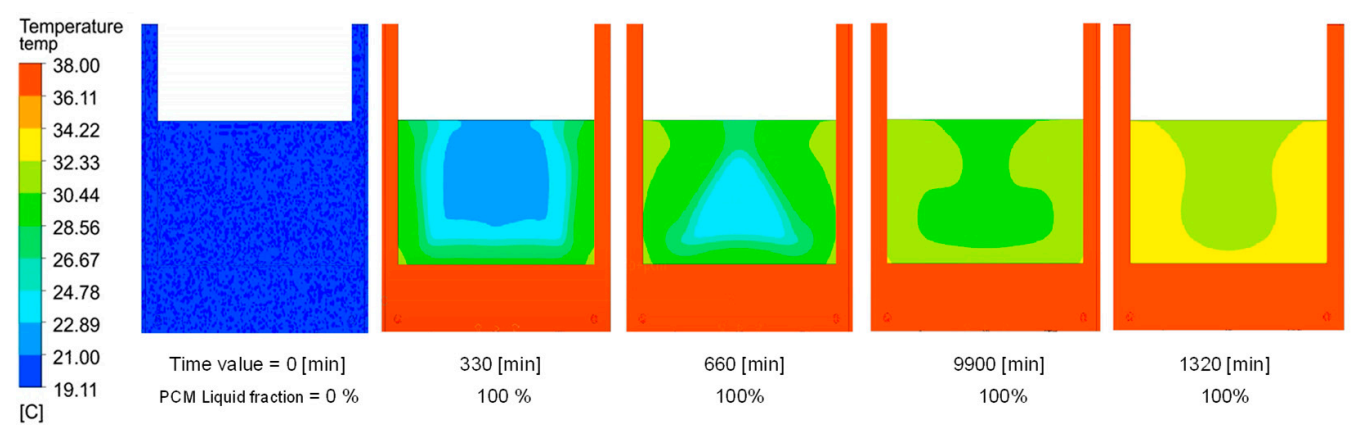

Figure 11. Case 3: Temperature and liquid fraction evolution during the charging process of PCM + water.
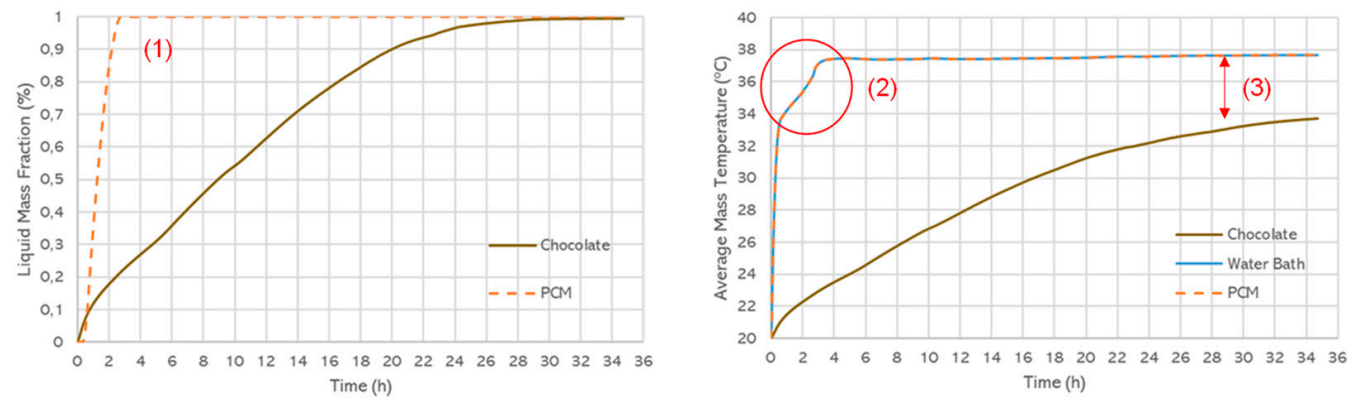

Figure 12. Case 3: Temperature (right) and liquid fraction (left) evolution during the charging process of PCM + water. The difference between the PCM and the water bath temperature curves is residual.

From Case 3 results it is possible to observe:

- The PCM charging period, with latent heat, ran between around $t=14 \mathrm{~min}(\approx 0.23 \mathrm{~h})$ and $t=75 \mathrm{~min}(1.25 \mathrm{~h})$, reaching over $99 \%$ of the liquid fraction of the PCM at the latter moment.

- Up to the value of the phase change temperature $\left(34^{\circ} \mathrm{C}\right)$, there was a linear increase in temperature in the set "water + PCM", consistent with the thermal storage in SHS. After reaching $34^{\circ} \mathrm{C}$, the temperature increase slowed down, as the PCM LHS started

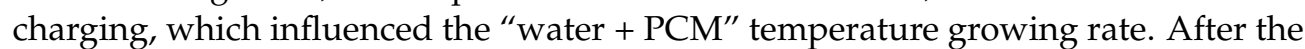
PCM was fully charged, the temperature of the "PCM + water" set returned to the initial linear growth with a shallower slope, representing a new period of SHS, which was maintained until reaching the temperature of the heat source.

- The difference between the temperature of the "water + PCM" set for the average temperature of the chocolate was justified by the thermal gradients within the chocolate mass. Although the temperature of the chocolate near the steel surface of the tank was close to the temperature inside the water jacket, it decreased towards the axis, with maximum gradients of $16^{\circ} \mathrm{C}$.

When comparing these results with those for Case 1 (see Figure 13, the main difference is the lack of inflection associated with the latent heat storage (region 2 in Figure 12). The temperature in the water jacket obeyed a linear evolution until $38^{\circ} \mathrm{C}$, since a constant thermal power was being considered. This temperature limit was achieved one hour earlier than in Case 3. However, this did not significantly change the average chocolate temperature evolution. There was a fast growth near the tank walls, with higher temperature gradients to the axis. These differences tended to vanish as the chocolate curve converged between the two cases. As a reference, at $26 \mathrm{~h}$ of charging, the liquid fraction of chocolate 
in Case 1 was $98.0 \%$ and the average temperature of the chocolate mass was $32.6{ }^{\circ} \mathrm{C}$, which was approximately the same as in Case 3 . These results suggest that although the PCM increased the storage capacity, initially slowing the temperature growth in the water jacket, this had no expression in the evolution of the temperature and state of the chocolate. This is a positive outcome for the established goals: the storage capacity was increased, absorbing more available thermal energy, without affecting the operation.
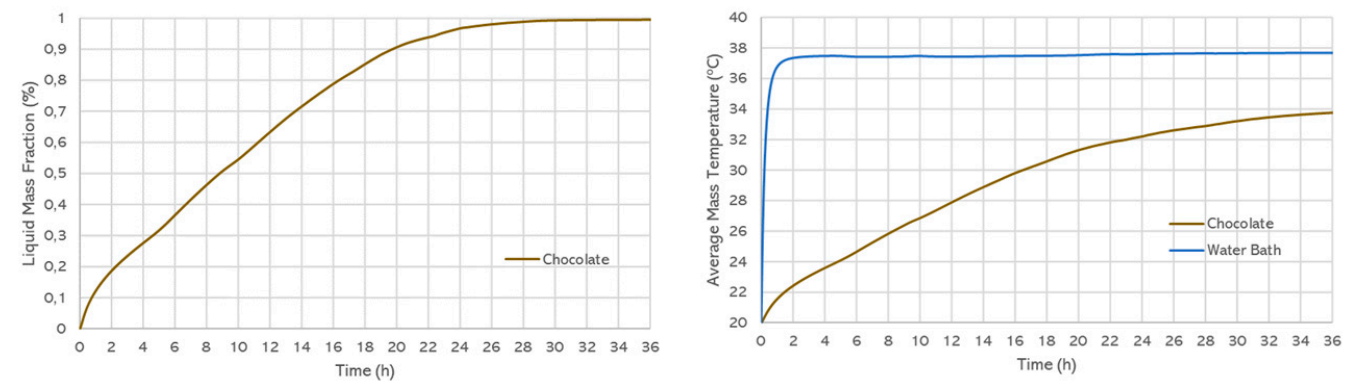

Figure 13. Case 1: Temperature (right) and liquid fraction (left) evolution during the charging process of water.

In Cases 2 (discharge of water) and 4 (discharge of water + PCM), the discharge line of the water jacket and the chocolate average temperature presented one main difference; see Figure 14. In Case 2, there was an exponential decrease towards room temperature, while in Case 4 there were three nearly linear segments with different slopes before, during, and after the solidification of the PCM. The temperature decrease was slower during the LHS discharge, which extended the operation period.
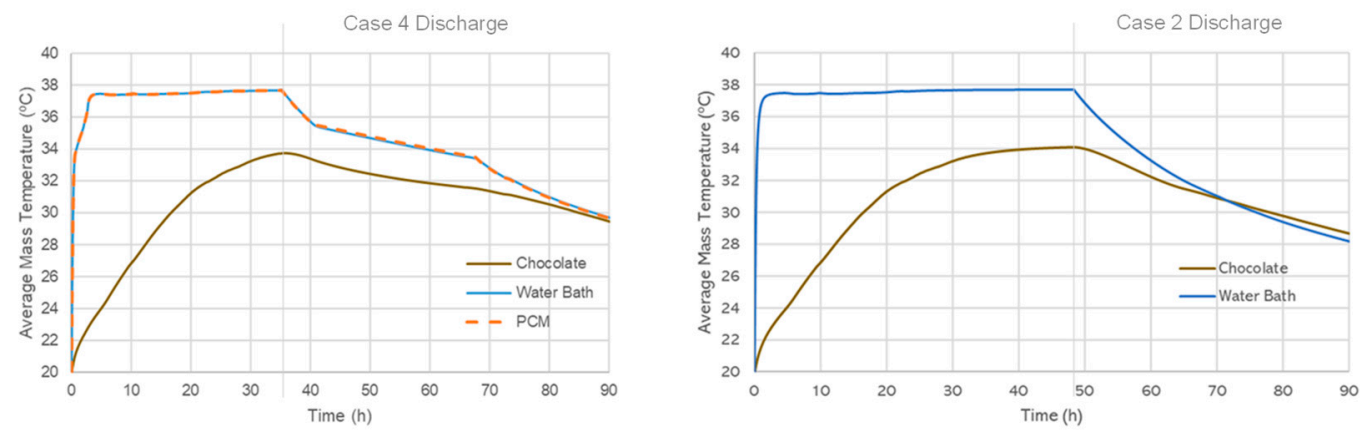

Figure 14. Case 4: Temperature evolution during the PCM + water discharging process (left) and Case 2: Temperature evolution during the water charging process (right).

A further simulation named Case 5, which was a variation of Case 3, with the inner tank filled with chocolate, was also carried out. It presented slower charge and discharge times and larger horizontal and vertical temperature gradients, with no other relevant differences, when compared to Case 3. This suggests that the chocolate level (60\% to 100\%) does not affect the operation paradigm, only the involved periods. Simulation results are shown in Figure 15.

\subsection{Experimental Results}

The experimental apparatus for testing the proposed TES/LHS concept was implemented over the tempering and mixing equipment described in Section 2.1 and shown in Figure 4 . 

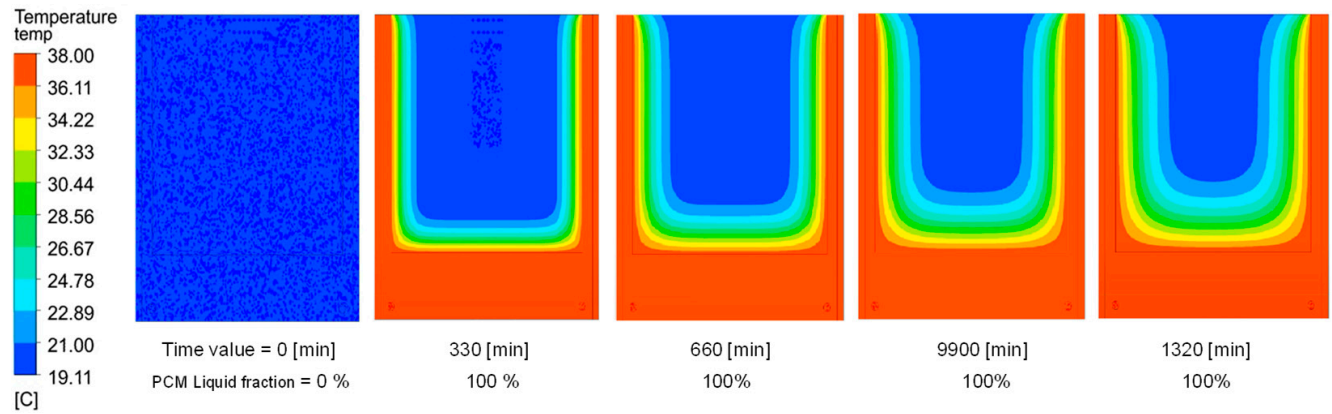

Figure 15. Case 5: Temperature and liquid fraction evolution during the charging process of PCM + water, with the chocolate level at $100 \%$.

\subsubsection{Monitoring Platform}

For monitoring and consolidating experimental data, an Internet of Things (IoT) modular architecture was developed over the Azure platform, using a Microcontroller Unit (MCU) PSOC 5LP (32 bits ARM, $80 \mathrm{MHz}$ ) from Cypress, integrating temperature and electric power sensors. The temperature sensors were attached to key points of the equipment, namely in the inner tank, in the outer tank, and between both; see Figure 16 . They were named T1 (water jacket temperature), T2 (inner tank inner wall temperature), T3 (inner tank lower temperature at the middle), T4 (inner tank upper temperature at the middle), and T5 (outer tank outer wall temperature).
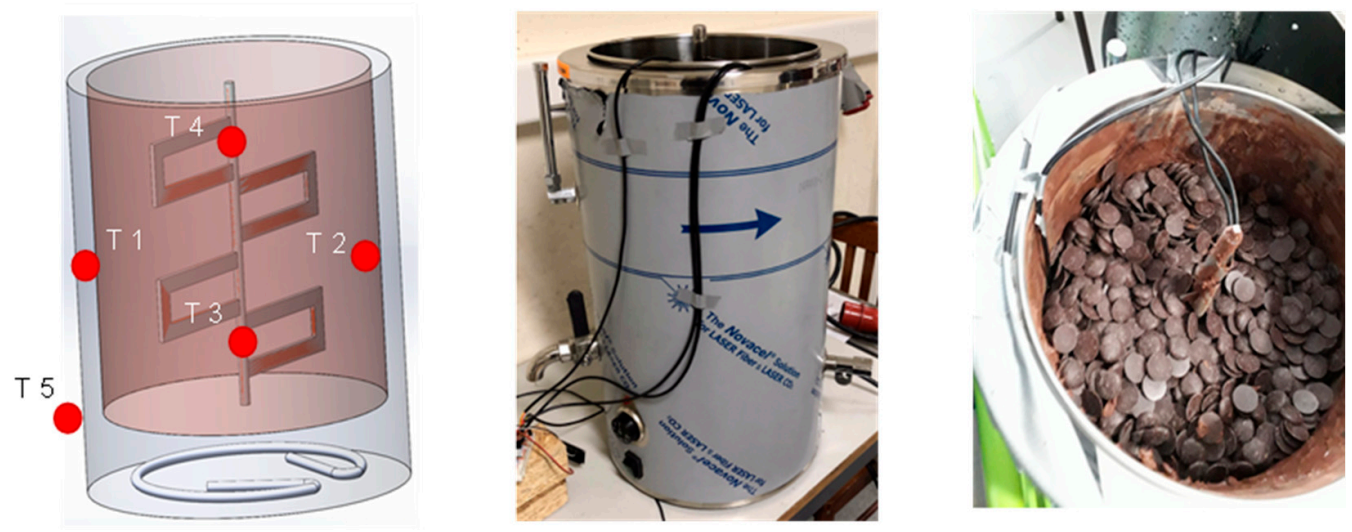

Figure 16. From left to right, location of the temperature sensors in the equipment; view of the cabling that connects the temperature sensors, T1 to T5, to the MCU; and chocolate pellets inside the inner tank.

In selecting the $\mathrm{MCU}$, its processing and data storage capacity were valued, as well as the availability of an open-source development platform and its interoperability with different technologies of data acquisition sensors. The platform was designed to respond to the specificity of the case study, but prepared to allow incremental growth, namely the integration of more or other types of sensors. The temperature sensors were selected according to their temperature ranges, but also to the versatility of the communication protocol. The model DS18B20 12-bit digital thermistor (one-wire) from Maxim Integrated was selected for its high stability, precision, and reduced dimensions. For monitoring the room temperature and humidity, the MPM station from Schneider Electric was used. The electric power consumed by the equipment was measured with the ACS71020 IC, from Allegro Microsystems, which monitors voltage, current, and power factor (since heat is provided by an electric resistor, the latter is unitary). To be able to collect temperature data on the inner tank shaft, it was necessary to remove the stirring arm. This option, also reflected in the simulation cases, enhanced the development of thermal gradients in the chocolate mass, but had no noticeable influence on the water jacket temperature or the PCM liquid fraction evolution. 
As a large amount of thermal energy is stored and released in a small temperature range around the PCM phase transition, it was important to investigate the PCM's cyclic stability [18] under operational conditions, as an influence factor in the experimental application longevity. To do so, as no appropriate testing devices were commercially available, the used PCM was first subjected to a set of non-automatic, manually induced, melting and solidification cycles, in a temperature range taking into account the conditions of the intended application, and then subjected to another set of melting and solidification cycles in an interval with a much higher temperature $\left(76^{\circ} \mathrm{C}\right)$. After that, the melting and solidification cycles under the conditions of the intended application were repeated. Although the number of testing cycles should be much larger than manually possible to perform in order to ensure a relevant scale for stronger conclusions on PCM's cyclic stability, no noticeable variations were detected in the cooling/heating rates between the first and the third set. The tests were carried out at an ambient temperature of around $16.4{ }^{\circ} \mathrm{C}$, with a relative humidity of $56 \%$. As already mentioned, the selected PCM was a hydrated salt (again, reference S34 from PCM Products) with a melting temperature of $34{ }^{\circ} \mathrm{C}$, encapsulated in sectioned bags built of flexible low-density polyethylene (LDPE), as already shown in Figure 7.

\subsubsection{Case Studies}

Eight different experiments were run involving the charge and discharge of the $11.5 \mathrm{~L}$ water jacket, with and without PCM, having the inner tank partially filled (60\%) with two distinct mixtures of granulated chocolate (density $1.26 \mathrm{~kg} / \mathrm{m}^{3}$ ). The characteristics of these mixtures were the following:

- $\quad$ Mixture 1: $15 \mathrm{~kg}$ of granulated chocolate $(11.9 \mathrm{~L})$, plus $2 \mathrm{~L}$ of milk. The total volume was $13.9 \mathrm{~L}$, corresponding to around $60 \%$ of the volume of the inner tank.

- Mixture 2: $12.5 \mathrm{~kg}$ of granulated chocolate $(9.9 \mathrm{~L})$, plus $6 \mathrm{~L}$ of milk. The total volume was $15.9 \mathrm{~L}$, corresponding to around $70 \%$ of the volume of the inner tank.

The different proportions of chocolate $(7.5 \mathrm{~kg} / \mathrm{L}$ of milk vs. $2.1 \mathrm{~kg} / \mathrm{L}$ of milk) were aimed at assessing the influence of milk on the performance of the charge/discharge process. It was later found that the impact was residual.

The experiments, named from \#1 to \#8, are described in Table 3. To compare the LHS and SHS storing performance, two temperature operation intervals were considered, namely from 34 to $38^{\circ} \mathrm{C}$ (SHS), and from 30 to $34^{\circ} \mathrm{C}$ (both SHS and LHS).

Table 3. Summary and denomination of the experiments.

\begin{tabular}{cc}
\hline Description & Denom. \\
\hline SHS charge, 11.5 L of water, tank at 60\%, chocolate mixture 1 & $\# 1$ \\
SHS discharge, 11.5 L of water, tank at 60\%, chocolate mixture 1 & $\# 2$ \\
SHS + LHS charge, 8 L water + 3.5 L PCM, tank at 60\%, chocolate mixture 1 & $\# 3$ \\
SHS + LHS discharge, 8 L water + 3.5 L PCM, tank at 60\%, chocolate mixture 1 & $\# 4$ \\
SHS charge, 11.5 L of water, tank at 60\%, chocolate mixture 2 & $\# 5$ \\
SHS discharge, 11.5 L of water, tank at 60\%, chocolate mixture 2 & $\# 6$ \\
SHS + LHS charge, 8 L water + 3.5 L PCM, tank at 60\%, chocolate mixture 2 & $\# 7$ \\
SHS + LHS discharge, 8 L water + 3.5 L PCM, tank at 60\%, chocolate mixture 2 & $\# 8$ \\
\hline
\end{tabular}

Before showing the experimental results, it is important to establish criteria for comparing these and the ones resulting from CFD simulations. It is possible to compare the experimental results among themselves, but it is not possible to directly compare the charging periods between the simulations and the experiments, because the heat source has different nature in the two scenarios. In the ANSYS simulation, a constant heating temperature of $38^{\circ} \mathrm{C}$ was considered, while in the experiments a heat source controlled by a thermostat, operated by on/off pulses of $3000 \mathrm{~W}$, was used. Although the heat source was turned off during the discharge period, the comparison of results during this period depends on the initial conditions. At the beginning of the discharge, the temperature in 
the water jacket is similar in all cases $\left(38^{\circ} \mathrm{C}\right)$, but the average temperature in the chocolate mass is not the same, due to the internal thermal gradients. However, this approximation is admitted in the analysis.

Some theoretical calculations should also be mentioned:

- $\quad$ The energy stored as sensible heat in $11.5 \mathrm{~L}$ of water (mass $m_{\text {water }}=11.5 \mathrm{~kg}$ ) when its temperature is increased from 16.4 to $38.0{ }^{\circ} \mathrm{C}$ (temperature variation $\Delta T=21.6^{\circ} \mathrm{C}$ ), considering an average specific heat for water $\bar{C}_{P}=4.13 \mathrm{~kJ} \mathrm{~kg}^{-1} \mathrm{~K}^{-1}$, is $Q_{\text {water }}=m_{\text {water }} \cdot \bar{C}_{P} \cdot \Delta T=1026 \mathrm{~kJ}$. This is the SHS corresponding to \#1,\#2, \#5 and \#6.

- Using the previous calculations, the energy stored in $8 \mathrm{~L}$ of water is $714 \mathrm{~kJ}$.

- $\quad$ The energy stored in the PCM depends on its mass $\left(m_{P C M}=7.35 \mathrm{~kg}\right.$, for a density $\left.\rho=2100 \mathrm{~kg} \cdot \mathrm{m}^{-3}\right)$, its phase change (melting) temperature $\left(T_{p c}=34{ }^{\circ} \mathrm{C}\right)$, the initial and final temperatures $\left(T_{i}\right.$ and $T_{f}$, respectively), the specific heat of its solid and liquid phases (assumed equal, with average values $\bar{C}_{s p}=\bar{C}_{l p}=2.1 \mathrm{~kJ} \mathrm{~kg}^{-1} \mathrm{~K}$ ), the fraction of its total mass that is melted (assumed $100 \%$, i.e., $\alpha_{m}=1$ ), and its phase change enthalpy $\left(\Delta H_{m}=140 \mathrm{~kJ} \mathrm{~kg}^{-1}\right)$. Previous values were already given in Section 3.2.1, and the total SHS and LHS is thus given by [11] $Q_{P C M}=m_{P C M} \cdot\left[\bar{C}_{s p} \cdot\left(T_{p c}-T_{i}\right)+\alpha_{m} \cdot \Delta H_{m}+\bar{C}_{l p} \cdot\left(T_{f}-T_{p c}\right)\right]=1362 \mathrm{~kJ}$.

Considering the already mentioned limitations, the main test results are presented and analyzed below:

1. With the introduction of 3.51 of PCM, corresponding to around $30 \%$ of the $11.5 \mathrm{~L}$ water jacket capacity, there was an increase of $1050 \mathrm{~kJ}(102 \%)$ in the stored energy, which was $1026 \mathrm{~kJ}$ (only with SHS), rising to $2076 \mathrm{~kJ}$ total (714 kJ as SHS, in $8 \mathrm{~L}$ of water, and $1362 \mathrm{~kJ}$ as LHS and SHS, in the PCM).

2. An increase in the charge and discharge periods was observed due to the PCM incorporation, which was due to the rise in the stored thermal energy. Comparing the discharges in \#2 (Figure 17) and \#4 (Figure 18), the PCM introduction allowed the increase in the discharge period $\left(38\right.$ to $30^{\circ} \mathrm{C}$ ) of $272 \%$ (in line with CDF simulation increase), extending the operation period above $30{ }^{\circ} \mathrm{C}$ in $8.6 \mathrm{~h}$, from $5.0 \mathrm{~h}$ to a total of $13.6 \mathrm{~h}$.

3. In both \#2 (Figure 17) and \#6 (Figure 19), only with SHS, with mixtures 1 and 2, respectively, during the discharge period $\left(38\right.$ to $\left.30^{\circ} \mathrm{C}\right)$, was it possible to observe a nearly steady linear reduction in the water jacket temperature. When comparing \#4 and \#8 (Figure 20) for LHS and SHS, it was possible to recognize a changing point with a lower rate of temperature decrease after $34^{\circ} \mathrm{C}$. The reductions were $42 \%$ in \#4 (mixture 1), and 43\% in \#8 (mixture 2), suggesting a residual dependence of the chocolate mixture used on the storage performance.

4. In addition to the increase in the storage capacity, which is essential for the development of a distributed storage architecture, the introduction of PCM led to increased thermal stability and a prolonged period of operation, lowering the rate of loss below the melting temperature $\left(34^{\circ} \mathrm{C}\right)$, from $1.30{ }^{\circ} \mathrm{C} / \mathrm{h}$ to $0.74{ }^{\circ} \mathrm{C} / \mathrm{h}$, as reference for the discharge in \#8.

5. In \#8, (SHS + LHS), the chocolate discharged at approximately the same rate as the PCM (respectively $0.73{ }^{\circ} \mathrm{C} / \mathrm{h}$ and $0.74{ }^{\circ} \mathrm{C} / \mathrm{h}$ ). This was different from $\# 6$ discharge (SHS), where the water cooled down faster than the chocolate, which started to provide heat for the water jacket (Figure 19, T2 > T1). Using the PCM, heat was not removed from the chocolate mix and therefore the working interval was extended by around $1 \mathrm{~h} 20$ of operation above $30{ }^{\circ} \mathrm{C}$ when compared to water (32\% more). This suggests that in the event of the full replacement of water by PCM, the period of operation above $30^{\circ} \mathrm{C}$ would be doubled.

6. Although it was expected to observe temperature gradients in the chocolate mass, they were enhanced by the removal of the stirring arm. In the two mixtures tested, vertical and horizontal gradients were observed during charging (hotter near the tank walls than in the center), whose direction was reversed during the discharge (hotter in 
the center of the tank than near the walls). During the charge, the highest temperature points were observed next to the inner tank wall and the base of the tank.

7. With the discharge and cooling of the chocolate mass, an inversion point was observed in which the temperature in the water jacket becomes lower than the temperature of the chocolate mass. This was more pronounced in SHS than in LHS cases, and it demonstrated that the chocolate mass had a larger capacity to retain heat than the water.

Although the PCM solution requires a higher energy consumption to complete the charge, this consumption is compensated by the extension of the operating cycle without the need to supply more heat. Thus, this would be relevant in the case where energy was provided by, for instance, a renewable source, such as solar thermal collectors. Moreover, it would add flexibility to the grid through distributed storage.

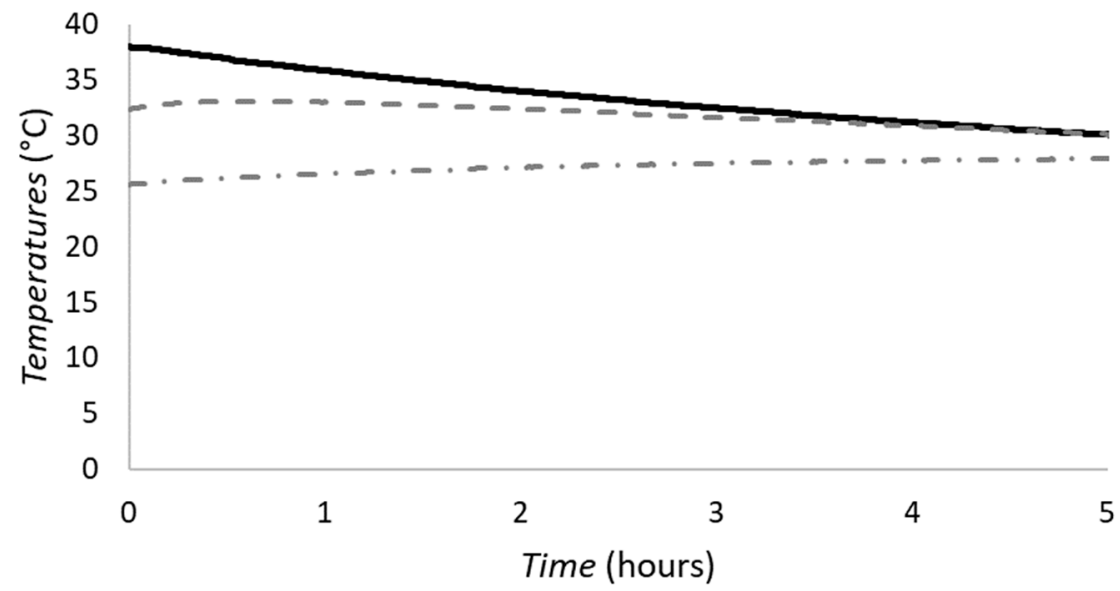

$$
\text { - } \mathrm{T} 1 \text { (Water) - - T2 - - - T4 }
$$

Figure 17. Heat discharging in $\# 2$, where only water is used for SHS, for chocolate mixture 1.

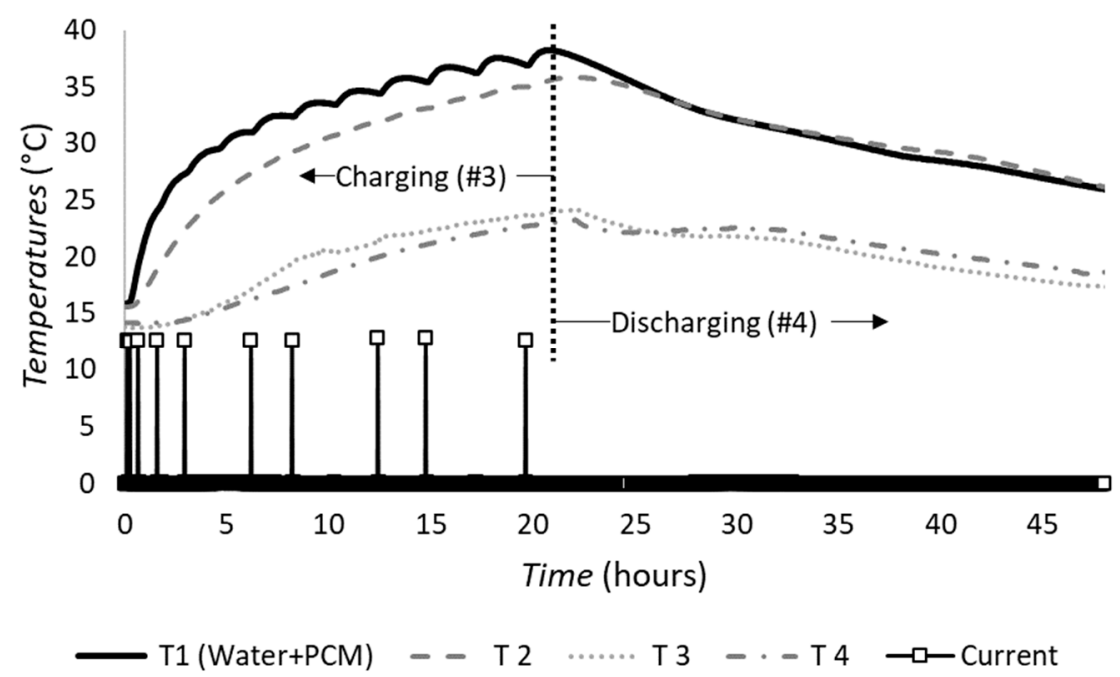

Figure 18. Heat charging (\#3) and discharging (\#4) when the PCM was used for LHS and SHS, for chocolate mixture 1. 


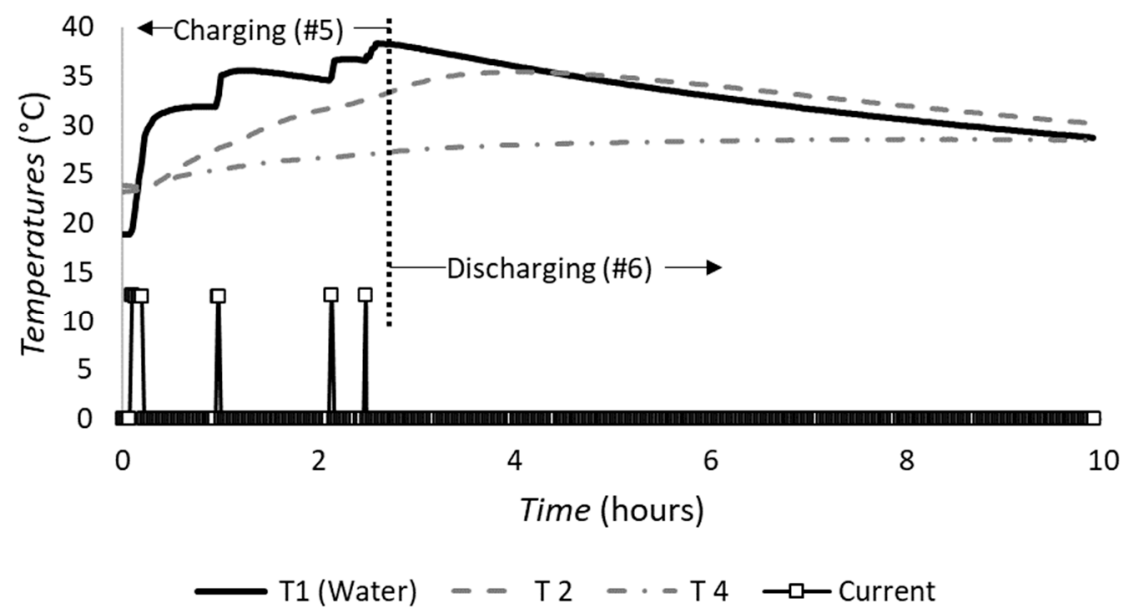

Figure 19. Heat charging (\#5) and discharging (\#6) when only water was used for SHS, for chocolate mixture 2.

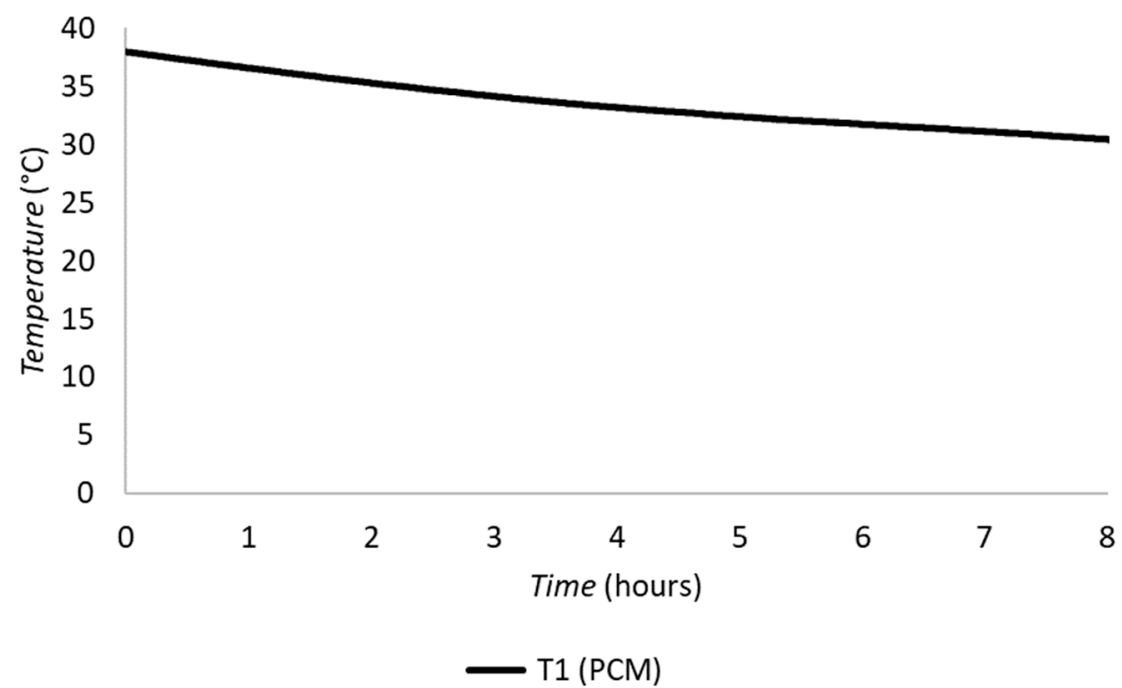

Figure 20. Detail of heat discharging (\#8), when the PCM was used for LHS and SHS, for chocolate mixture 2 .

One major practical drawback in the experimental tests was the degradation of the PCM encapsulation. As mentioned, the PCM was encapsulated in sectioned tablets built on flexible LDPE. This option proved to be adequate for the easy upgrade of equipment, which is an important vector for the dissemination of results. However, the option for a plastic enclosure, which is a cheap and flexible material, showed technical limitations. In addition to the degradation of the compartmentalization seals (see Figure 21, left), with the change in phase there was a deformation of the PCM bags, which molded to the walls of the inner tank. This process complicated the PCM removal, for instance, for maintenance purposes, tearing most of the wrappers, limiting their reuse (see Figure 21, right). With a view to maintaining the geometry, it will be important in future work to consider different encapsulation materials, such as aluminum foil. 

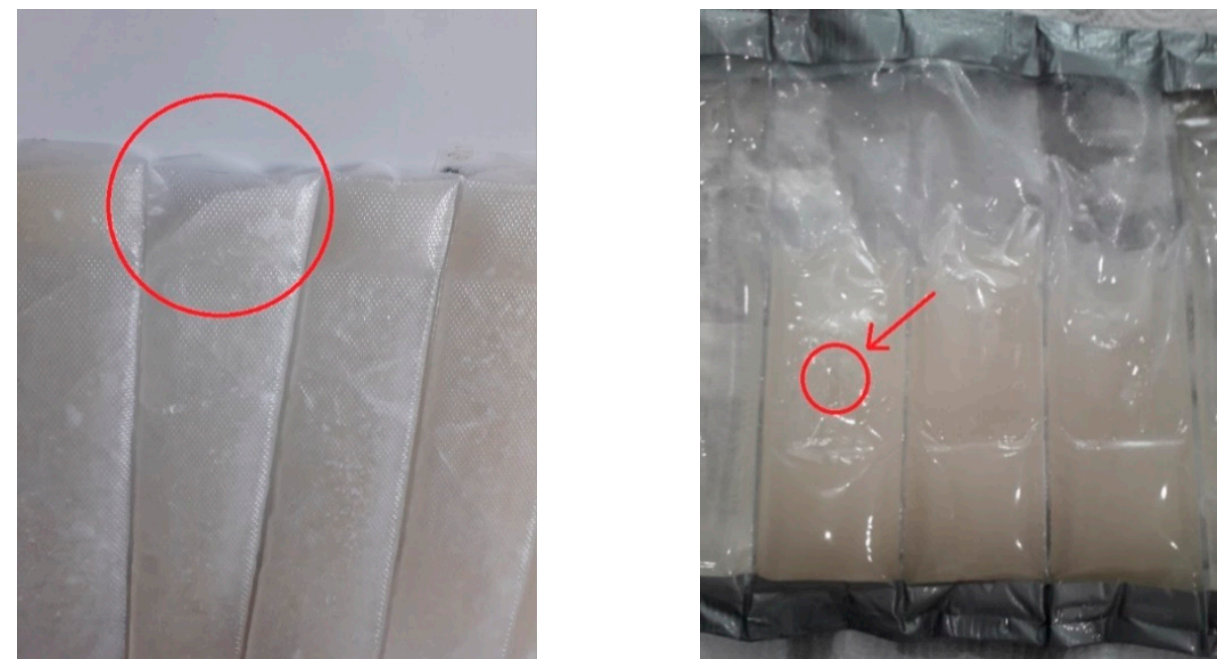

Figure 21. From left to right, detail of the degraded encapsulation of the PCM; PCM after cold withdrawn, in solid-state, consolidated at the base of the encapsulation, with points of rupture of the encapsulation.

\section{Discussion}

The major design aspects of a TES/LHS solution have been previously described, using PCMs in a distributed architecture on equipment representing a specific agroindustrial process cluster (water jacket). Experimental and simulated results were compared to demonstrate the added value of this paradigm, which allows for higher storage capacity and flexibility when compared to SHS.

The simulation model was validated as a "proof of concept" by the results of the experimental tests, paving the way for a new planning tool for this equipment cluster, which would thus become more efficient. Despite the previously mentioned limitations, based on the comparison between experimental measurements and the results of numerical simulations, it was possible to identify several agreements between both:

1. It was possible to observe patterns of temperature evolution in the experimental tests (charging and discharging scenarios) in line with what was predicted by the CFD simulations.

2. In the experimental charge and discharge scenarios with PCM, three distinct rates of temperature variations were identified, converging with the storage periods in SHS, (SHS + LHS) and again SHS, in line with what was predicted by numerical simulations.

3. Although the total discharge times from CFD and experimental tests were not comparable, they both presented similar improvements in operating times resulting from the integration of the PCM, respectively $175 \%$ and $172 \%$, when compared with using only water.

4. The numerical model and the experimental tests also converged in the formation of temperature gradients in the chocolate mass during the charge period, and in the inversion of direction during the discharge process.

As such, both the simulation and experimental processes demonstrated, in the first place, the possibility of incorporating a PCM directly into the structure of productive equipment, partially replacing the use of water as a source for thermal storage. Secondly, it was shown that the replacement of $3.5 \mathrm{~L}$ of water by the same volume of S34 PCM represented an increase of $1050 \mathrm{~kJ}(330 \%)$ of storage capacity in the same volume, and $102 \%$ in the combined set (3.5 L PCM $+8 \mathrm{~L}$ water). Once the PCM was charged, the gain boosted by the increase in storage capacity was directly reflected in the increase in the period of operation of the equipment in autonomy (without power supply), keeping the chocolate for $1.2 \mathrm{~h}(32 \%)$ more of operation above $30{ }^{\circ} \mathrm{C}$. In this work, a PCM selection methodology was presented, with valuation criteria based on the adequacy of the thermophysical properties 
of the materials for the operation requirements and on the impact on the exploration and maintenance processes, the cost and availability of materials, the main risk factors, and the life cycle impact. This study also addressed the importance of the encapsulation strategy, namely in terms of the choice of materials and geometry, for the mitigation of risks, maximization of thermal conductivity and heat exchange, and the longevity of the solution.

The possibility of increasing the storage capacity per $\mathrm{kg}$ of PCM was demonstrated when compared to the same ratio of conventional schemes using, for example, water for SHS. This foresees the potential of changing from centralized SHS to distributed LHS solutions.

The need for a seamless technological transition that does not compromise previous investments in the existing technological park, if feasible, must be addressed on a case-bycase basis, similarly to for the approach to the cluster of equipment operating under the model of "water jacket", to adequately answer the specifics of the operating temperature ranges and geometries of the structures of the targeted equipment. Although gains can be exploited at the level of isolated equipment, as in the case study, it is important to realize that these good indicators do not replace the need for scale to obtain commercially viable solutions. It is therefore important to broaden the spectrum of equipment that can incorporate PCMs in order to achieve an aggregated storage capacity per group of equipment, which competes with the energy storage values of current TES/SHS solutions.

\subsection{Financial and Economic Analysis}

In this subsection a methodology for financial and economic analysis is presented and carried out, aiming at assessing the prospective viability of the proposed concept. The following assumptions are considered:

- There are no costs with the operation and maintenance (O\&M) of the PCM during the analyzed periods, meaning that this can be included with the current O\&M costs of the prospective user;

- There is no need to replenish the PCM in the analyzed periods;

- The energy required to charge the PCM is originated in renewable sources (e.g., solar thermal), thus is free;

- The costs of implementing the previous renewable plant are not accounted for, meaning that it is considered already available;

- The costs of integrating the PCM in the industrial equipment are neglected, i.e., they are already included in the operation costs of the user.

For the financial analysis, the following values are considered, either obtained from previous results or market prospection:

- Water used as a sensible heat storage medium has an energy storage density of $89.2 \mathrm{~kJ} / \mathrm{L}$;

- The PCM used for sensible and latent heat storage has an energy storage density of $389.1 \mathrm{~kJ} / \mathrm{L}$;

- The PCM cost is $C_{P C M}=80.00 € / \mathrm{L}$;

- The average tariff of electrical energy in half-peak periods (typically corresponding to solar production) is $T_{E}=0.15 € / \mathrm{kWh}$.

There is, therefore, an increase in the storage capacity of $\Delta E_{v}=389.1-89.2=299.9 \mathrm{~kJ} / \mathrm{L}$ provided by the PCM, or $0.0833 \mathrm{kWh} / \mathrm{L}$.

The following parameters are defined as:

- $\quad N$, the number of yearly cycles of the system (i.e., charge and discharge cycles of the storage medium);

- $Y$, the number of operating years of the PCM, without its replenishment, corresponding to at least the considered investment horizon. 


\subsubsection{Financial Analysis}

The following cost is avoided at the end of the operating years (corresponding to the cost of the energy that was not imported from the electrical grid), in $€ / L$ :

$$
C_{e}=\Delta E_{v} \cdot T_{e} \cdot Y \cdot N=0.0125 \cdot Y \cdot N \text {. }
$$

In a financial analysis, for the PCM to be viable, the condition $C_{e}<C_{P C M}$ must be verified. The dependence of $C_{e}$ with $\mathrm{Y}$ and $\mathrm{N}$ is shown in Figure 22. Values below the considered $C_{P C M}$, namely $80.00 € / L$, are, thus, viable.

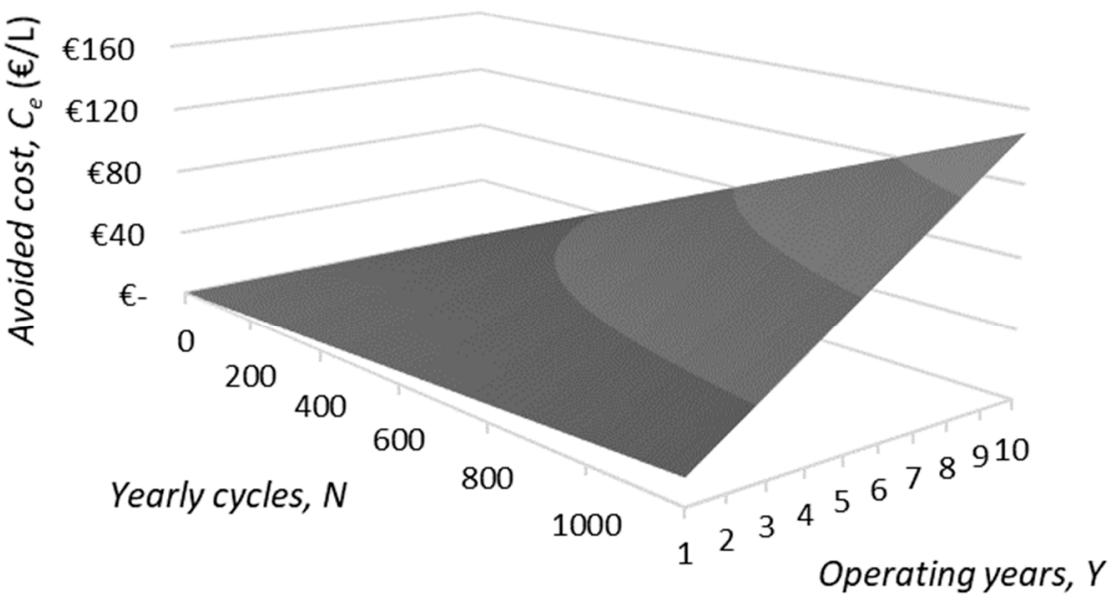

Figure 22. Dependence of the total avoided cost with the integration of PCM, $C_{e}$, with the number of early cycles of charge and discharge, $N$, and the operating years, $Y$. For the current example, values below $80 € / \mathrm{L}$ are viable.

On the other hand, the cost of the PCM, $C_{P C M}$, was obtained from a manufacturer and did not consider the influence of large-scale acquisition, nor its prospective decrease as this technology is disseminated. A sensitivity analysis for $C_{e}$ is presented in Figure 23, considering $C_{P C M}=70$ and $C_{P C M}=60 € / \mathrm{L}$, where the curves correspond to the transition surface from viability to non-viability of the proposed concept.

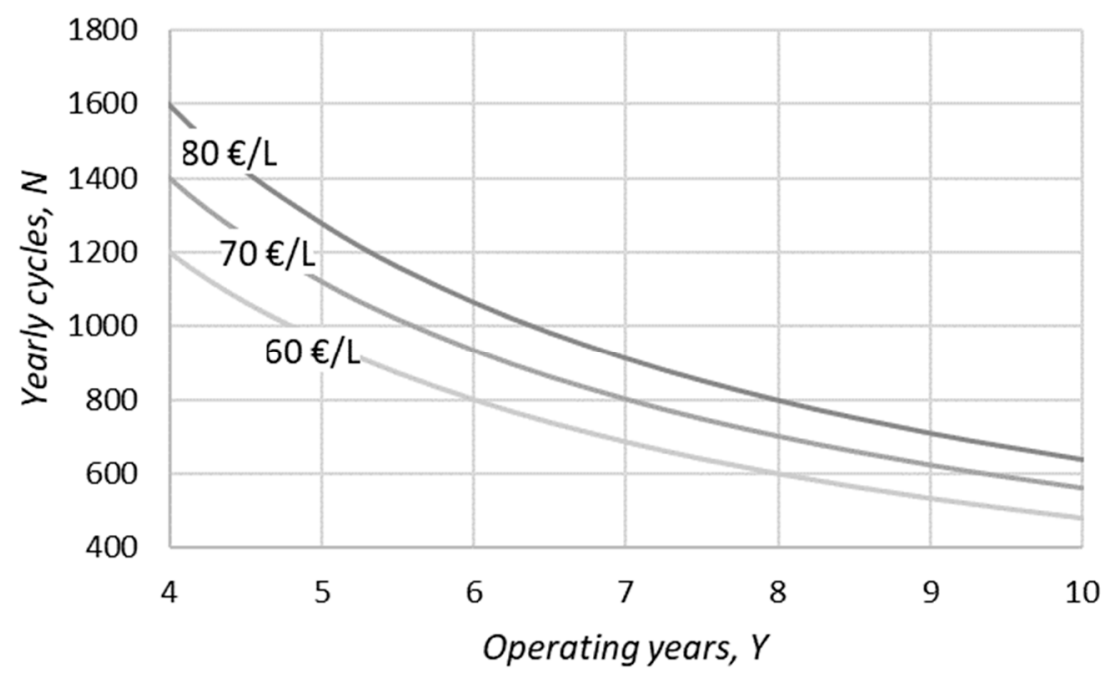

Figure 23. Results of the sensitivity analysis for the avoided cost, $C_{e}$, with the cost of the PCM, $C_{P C M}$. For instance, if 8 years of operation are considered, the concept is financially viable for, at least, around 800, 650, and 600 charge and discharge yearly cycles, corresponding to, respectively, 80, 70, and $60 € / \mathrm{L}$ of PCM cost. 


\subsubsection{Economic Analysis}

For the economic analysis, the Net Present Value $(N P V)$ and the Internal Rate of Return $(I R R)$ indicators are used. These are defined as

$$
\begin{aligned}
& N P V=\sum_{t=1}^{H} \frac{C F_{t}}{(1+r)^{t}}-I_{0} \\
& \sum_{t=1}^{H} \frac{C F_{t}}{(1+I R R)^{t}}-I_{0}=0,
\end{aligned}
$$

where $C F_{t}$ is the cash flow (balance of revenue and expense) in year $t, r$ is the discount rate (return possible to obtain in alternative investments), $H$ is the considered investment horizon, and $I_{0}$ is the initial investment. A positive NPV means a prospectively profitable investment. It is considered that the initial investment is fully executed at $t=0$.

For the example of $C_{P C M}=60 € / \mathrm{L}$, considering $r=6 \%, H=15$ years, $T_{E}=0.15 € / \mathrm{kWh}$, and $N=600$ prospective cycles per year, $N P V=12.82 € / \mathrm{L}$, which means a viable project. The net present accumulated cash flows are shown in Figure 24, where the payback period, considering net present values, is 12 years (the simple payback period, with simple, financial cash flows considered, was obtained at the end of the 8 th year). $I I R=9.1 \%$, more than $50 \%$ above $r$, was also obtained, which demonstrates the quality of the investment, under the previous assumptions.

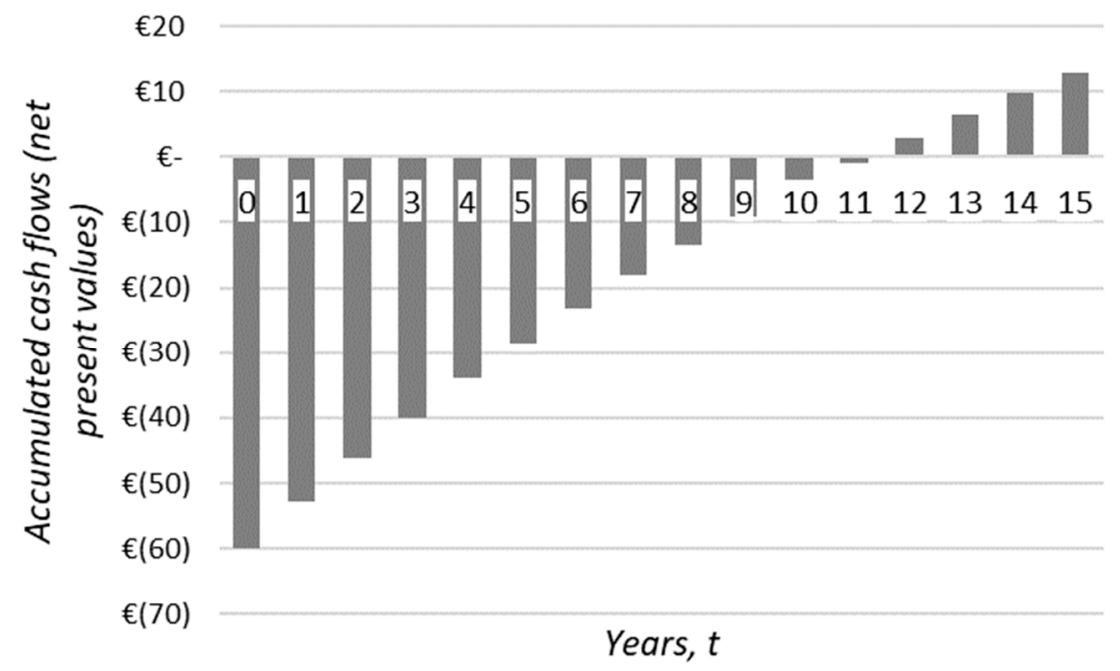

Figure 24. Accumulated cash flows, considering their net present values. The payback is obtained at year 12 , when values become positive.

\subsection{Future Work}

Besides the development of a PCM incorporation and encapsulation strategy, future work will consist of developing the thermal energy recovery and/or renewable integration circuits, to replace electrical energy supplied by the grid. The valorization of the flexibility enhanced by the TES/LHS solution requires integration with energy sources whose potential is still not fully exploited in the agroindustry. These could consist, as an example, in the recovery of IWH, as well as in the integration of renewable technologies. Among the latter, the option for solar thermal energy is envisaged, as it allows PCMs to be charged directly by heat exchange, also increasing the flexibility provided to the electrical grid. These two aspects, the diversification of equipment eligible for incorporation of PCMs and the development of energy recovery systems, will be the focus of subsequent work. 


\begin{abstract}
Author Contributions: Conceptualization, C.S. and J.M.-P.; methodology, C.S., J.M.-P. and F.R.; software, C.S., J.P. and D.F.; validation, J.M.-P., L.C., F.R., T.J. and P.F.; formal analysis, C.S., J.P. and D.F.; investigation, C.S., J.P., D.F. and J.M.-P.; resources, J.M.-P., F.R., J.P.O., L.C., T.J. and P.F.; data curation, J.M.-P., F.R., J.P.O., L.C. and J.P.; writing-original draft preparation, C.S. and J.M.-P.; writing-review and editing, L.C., J.P., F.R., J.P.O., T.J. and P.F.; visualization, C.S., J.P., D.F., T.J. and P.F.; supervision, J.M.-P., F.R. and J.P.O.; project administration, C.S. and J.M.-P.; funding acquisition, J.M.-P. All authors have read and agreed to the published version of the manuscript.
\end{abstract}

Funding: This work was supported by FCT (Fundação para a Ciência e a Tecnologia), within the scope of Research Unit CTS, Centre of Technology and Systems, with the reference UIDB/00066/2020.

Data Availability Statement: Not applicable.

Conflicts of Interest: The authors declare no conflict of interest.

\title{
References
}

1. Colier, P.; Page, J. Industrial Development Report 2009: Breaking In and Moving Up: New Industrial Challenges for the Bottom Billion and the Middle-Income Countries; United Nations Industrial Development Organization (UNIDO): Vienna, Austria, 2009.

2. Konig, G.; da Silva, C.A.; Mhlanga, N. Enabling Environments for Agribusiness and Agro-Industries Development: Regional and Country Perspectives; Food and Agriculture Organization of the United Nations (FAO): Rome, Italy, 2013.

3. Muller, D.C.A.; Marechal, F.M.A.; Wolewinski, T.; Roux, P.J. An energy management method for the food industry. Appl. Therm. Eng. 2007, 27, 2677-2686. [CrossRef]

4. Policy Brief: The Case for Energy-Smart Food Systems; Food and Agriculture Organization of the United Nations (FAO): Rome, Italy, 2011.

5. Latini, A.; Viola, C.; Scoccianti, M.; Campiotti, C.A. Efficient Fruit and Vegetables Processing Plants: Handbook; TESLA Project Report IEE/12/758/SI2.644752; TESLA: Austin, TX, USA, 2014.

6. Miró, L.; Gasia, J.; Cabeza, L.F. Thermal energy storage (TES) for industrial waste heat (IWH) recovery: A review. Appl. Energy 2016, 179, 284-301. [CrossRef]

7. Sinsel, S.R.; Riemke, R.L.; Hoffmann, V.H. Challenges and solution technologies for the integration of variable renewable energy sources-A review. Renew. Energy 2020, 145, 2271-2285. [CrossRef]

8. Chen, X.; Zhang, Z.; Qi, C.; Ling, X.; Peng, H. State of the art on the high-temperature thermochemical energy storage systems. Energy Convers. Manag. 2018, 177, 792-815. [CrossRef]

9. Cabeza, L.F. Advances in Thermal Energy Storage Systems; Elsevier: Amsterdam, The Netherlands, 2015.

10. Sarbu, I.; Sebarchievici, C. A Comprehensive Review of Thermal Energy Storage. Sustainability 2018, 10, 191. [CrossRef]

11. Sharma, A.; Tyagi, V.V.; Chen, C.R.; Buddhi, D. Review on thermal energy storage with phase change materials and applications. Renew. Sustain. Energy Rev. 2009, 13, 318-345. [CrossRef]

12. Su, W.; Darkwa, J.; Kokogiannakis, G. Review of solid-liquid phase change materials and their encapsulation technologies. Renew. Sustain. Energy Rev. 2015, 48, 373-391. [CrossRef]

13. Monforti-Ferrario, F.; Dallemand, J.; Pascua, I.; Motola, V.; Banja, M.; Scarlat, N.; Medarac, H.; Castellazzi, L.; Labanca, N.; Bertoldi, P.; et al. Energy Use in the EU Food Sector: State of Play and Opportunities for Improvement; Publications Office of the European Union: Luxembourg, 2015.

14. Garcia, N.P.; Vatopoulos, K.; Riekkola, A.K.; Lopez, A.P.; Olsen, L. Best Available Technologies for the Heat and Cooling Market in the European Union; Publications Office of the European Union: Luxembourg, 2012.

15. Li, Z.; Lu, Y.; Huang, R.; Chang, J.; Yu, X.; Jiang, R.; Yu, X.; Roskilly, A.P. Applications and technological challenges for heat recovery, storage and utilisation with latent thermal energy storage. Appl. Energy 2020, 283, 116277. [CrossRef]

16. Ure, Z.; Mashare, M. Phase change material based passive cooling systems design principal and global application examples. In Proceedings of the 12th International Conference on Sustainable Energy technologies (SET-2013), Hong-Kong, China, 26-29 August 2013.

17. Pielichowska, K.; Pielichowski, K. Phase change materials for thermal energy storage. Prog. Mater. Sci. 2014, 65, 67-123. [CrossRef]

18. Oró, E.; de Gracia, A.; Castell, A.; Farid, M.M.; Cabeza, L.F. Review on phase change materials (PCMs) for cold thermal energy storage applications. Appl. Energy 2012, 99, 513-533. [CrossRef]

19. Xu, H.; Sze, J.Y.; Romagnoli, A.; Py, X. Selection of phase change material for thermal energy storage in solar air conditioning systems. Energy Procedia 2017, 105, 4281-4288. [CrossRef]

20. Zsembinszki, G.; Fernández, A.G.; Cabeza, L.F. Selection of the appropriate phase change material for two innovative compact energy storage systems in residential buildings. Appl. Sci. 2020, 10, 6. [CrossRef]

21. Nienborg, B.; Gschwander, S.; Munz, G.; Fröhlich, D.; Helling, T.; Horn, R.; Weinläder, H.; Klinker, F.; Schossig, P. Life cycle assessment of thermal energy storage materials and components. Energy Procedia 2018, 155, 111-120. [CrossRef]

22. Kenisarin, M.M.; Mahkamov, K.; Costa, S.C.; Makhkamova, I. Melting and solidification of PCMs inside a spherical capsule: A critical review. J. Energy Storage 2020, 27, 101082. [CrossRef]

23. Sarani, I.; Payan, S.; Nada, S.A.; Payan, A. Numerical investigation of an innovative discontinuous distribution of fins for solidification rate enhancement in PCM with and without nanoparticles. Appl. Therm. Eng. 2020, 176, 115017. [CrossRef] 
24. Mahdi, J.M.; Nsofor, E.C. Solidification enhancement of PCM in a triplex-tube thermal energy storage system with nanoparticles and fins. Appl. Energy 2013, 211, 975-986. [CrossRef]

25. Jones, W.P.; Launder, B.E. The prediction of laminarization with a two-equation model of turbulence. Int. J. Heat Mass Transf. 1972, 15, 301-314. [CrossRef] 